\title{
A kinetic approach to granular gases
}

\author{
A. Puglisi ${ }^{1}$, V. Loreto ${ }^{2,3,1}$, U. Marini Bettolo Marconi ${ }^{4,5}$ and A. Vulpiani ${ }^{1}$ \\ (1) Dipartimento di Fisica, Università La Sapienza, Piazzale A. Moro 2, 00185 Roma, Italy and \\ Istituto Nazionale di Fisica della Materia, Unità di Roma \\ (2) P.M.M.H., Ecole Supérieure de Physique et Chimie Industrielles, 10, rue Vauquelin, 75231 \\ Paris, France \\ (3) ENEA Research Center, Località Granatello C.P. 3280055 Portici, Napoli, Italy \\ (4) Dipartimento di Matematica e Fisica, Università di Camerino, Via Madonna delle Carceri, \\ I-62032, Camerino, Italy and Istituto Nazionale di Fisica della Materia, Unità di Camerino
}

(5) Istituto Nazionale di Fisica Nucleare, Sezione di Perugia

(November 12, 2017)

\begin{abstract}
We address the problem of the so-called "granular gases", i.e. gases of massive particles in rapid movement undergoing inelastic collisions. We introduce a class of models of driven granular gases for which the stationary state is the result of the balance between the dissipation and the random forces which inject energies. These models exhibit a genuine thermodynamic limit, i.e. at fixed density the mean values of kinetic energy and dissipated energy per particle are independent of the number $N$ of particles, for large values of $N$. One has two regimes: when the typical relaxation time $\tau$ of the driving Brownian process is small compared with the mean collision time $\tau_{c}$ the spatial density is nearly homogeneous and the velocity probability distribution is gaussian. In the opposite limit $\tau \gg \tau_{c}$ one has strong spatial clustering, with a fractal distribution of particles, and the velocity probability distribution strongly deviates from the gaussian one. Simulations performed in one and two dimensions under the Stosszahlansatz Boltzmann approximation confirm the scenario. Furthermore we analyze the instabilities bringing to the spatial and the velocity clusterization. Firstly, in the framework of a mean-field model, we explain how the existence of the inelasticity can bring to a spatial clusterization; on the other side we discuss, in the framework of a Langevin dynamics treating the collisions in a mean-field way, how a non-gaussian distribution of velocity can arise. The comparison between the numerical and the analytical results exhibits an excellent agreement.
\end{abstract}

PACS: 81.05.Rm, 05.20.Dd, 05.40.+j

\section{INTRODUCTION: HYDRODYNAMICS AND GASES}

Granular systems (sand, powders, seeds, cements, etc.) have been extensively studied, in the last two decades, by means of analytical investigations, experiments and computer 
simulations. The rich and intriguing phenomenology is well known to engineers and the need of a better comprehension of granular behaviors is widely recognized in applied sciences as well as in theoretical physics. A quite exhaustive review may be found in [1]. Problems in granular systems are roughly divided in quasi-static (sand piles, distribution of static forces, compaction, fractures propagation, etc.) and dynamical ones (all kind of flows, convection and segregation, pattern formation, fluidized beds, etc.). In the latter class, large collections of inelastic particles are involved in fluid-like rapid dynamics, therefore the hydrodynamics approach seems to be the natural one. The main granular hydrodynamics theories are reviewed in [2]: they are all based on non-equilibrium conservation laws [3] for mass, momentum and energy:

$$
\begin{gathered}
\frac{d \rho}{d t}+\rho \nabla \cdot \mathbf{u}=\mathbf{0} \\
\rho \frac{d \mathbf{u}}{d t}=-\nabla \cdot \hat{\tau}+\rho \mathbf{g} \\
\frac{1}{2} \rho \frac{d T}{d t}=-\nabla \cdot \mathbf{q}+\hat{\tau}: \nabla \mathbf{u}-\Gamma
\end{gathered}
$$

where $\rho$ is the local density, $\mathbf{u}$ is the local velocity vector, $d / d t=\partial / \partial t+\mathbf{u} \cdot \nabla$ is the Lagrangian derivative, $\hat{\tau}$ is the total stress tensor $\left(\hat{\tau}=\hat{\tau}_{s}+\hat{\tau}_{c}\right.$, as transport of momentum has two contributions: a "streaming" term and a "collisional" one), $\mathrm{g}$ is the body-force vector (gravity or else), $T$ is the so-called granular temperature $\left.T=<(\mathbf{u}-<\mathbf{u}\rangle)^{\mathbf{2}}\right\rangle, q$ is the flux vector of "granular heat", $\hat{\tau}: \nabla \mathbf{u}$ is the tensorial product for the granular-temperature generation by shear work and $\Gamma$ is the sink term due to dissipation into thermodynamic heat (i.e., energy lost in collisions). The existing approaches differ in the constitutive relations that make $\tau, q$ and $\Gamma$ depend on the other properties $\nabla \mathbf{u}, \rho, T$ and on all the parameters of the system. Apart some heuristic relations (see, for example, [4] or [5]), there are many calculations based on the kinetic theory of nonuniform dense gases [6], making some assumption on the form of the single particle distribution function $f(\mathbf{x}, \mathbf{v}, \mathbf{t})$, that is the solution of the Boltzmann-Enskog kinetic equation. Before [7], and the almost simultaneous [8], all the theories had assumed a Maxwellian velocity distribution, but the nonzero off diagonal components in the streaming stress tensor (strongly apparent at low solid fractions) indicate the need of a correction to Maxwellian distribution. In [7] and [8] accurate predictions of streaming stresses, in the case of slightly inelastic and slightly rough smooth spheres, are obtained. In [7] and [8] and in successive studies (see [2]), a certain degree of energyequipartition breaking is assumed, considering two different temperatures for translational and rotational degrees of freedom, respectively.

The increasing power of computers awakened an interest in "granular gases" simulations, that is the investigation of kinetics of granular systems far from close-packing, e.g. a granular phase diagram has been proposed in [9] to clarify different regimes and distinguish between a gas-like phase and two different high-density phases. Results from these simulations have been compared to previous granular hydrodynamics predictions, showing a disagreement in various aspects. 
Many simulations have been performed in the "cooling" situation ( [10], [11], 112], [13], |14], 15]): the particles evolve with no external forcing, dissipating in collisions all initial energy. Hydrodynamic predicts a time-scaling of granular temperature $T \sim t^{-2}$ under the assumption of Maxwellian velocities at all times, but strong departures from this law are observed when (at fixed volume) the number of particles grows or when the restitution coefficient $r$ decreases [11]. When $N(1-r)>>1$ [12] (where $N$ is the number of particles) it is found that the clustering instability (this can be derived 10 from Jenkins \& Richman hydrodynamics [8]) may degenerate in the so-called inelastic collapse, as particles may be trapped in a sequence of infinite collisions in a finite time (i.e. a divergence of collision rate). Inelastic collapse is found in one-dimensional and two-dimensional [13] simulations. Furthermore, equipartition between rotational and translational energy is found to be broken in cooling kinetics (see [15] and references therein).

Strong equipartition breaking is found in another class of models, that of driven granular gases, where the dissipation of energy due to collisions is balanced by an external source (in realistic situations one has to vibrate or shake a granular system, to keep it alive). We could divide these models in two sub-classes: elitary and democratic models, referring to the quantity of particles receiving energy from the external source. In elitary (one dimensional [16], [17] and two-dimensional [17]) models a wall of the container is the unique energy source, therefore there are few particles (just one, in 1d) that transport energy from the source to the rest of the system. In democratic models (one dimensional [18], [19] and two-dimensional [20]) all particles receive energy, by mean of a Brownian-like random velocity kick at every time step. The model we propose in this paper belongs to this last sub-class.

Recently the Boltzmann-Enskog equation for granular kinetic (cooling or driven) has been analyzed, showing that the velocity distribution is expected not to be Maxwellian. Esipov and Pöschel [21] have found, for cooling inelastic hard spheres, exponential tails while van Noije and Ernst [22] have obtained the same tails in the cooling regime and $\sim \exp \left(-A v^{3 / 2}\right)$ tails in the (democratic) driven regime.

A non-Maxwellian behavior has been, very recently, observed experimentally in a vertically driven granular bed [23]. The measured velocity distribution in such experiment is in very good agreement with the results of our simulations.

To conclude this brief introduction, one has to remember that, in modeling granular gases, the oversimplifying criterion is, sometimes, misleading. Brilliantov et al. have shown [24] that the universally accepted picture of fixed restitution coefficient is far from being obvious and that the behavior of granular gases (self diffusion, as an example) may change drastically if this coefficient is taken dependent on the impact-velocity. McNamara and Luding [25] have recently stressed the importance of keeping into account rotational degrees of freedom as well translational ones, and that of the particle-wall dissipation. Furthermore, the relevance of choosing the boundary energy source and the possibility of considering soft particles instead of hard grains have been investigated by Geisshirt et al [26].

In section 1 detailed results of simulations of a one-dimensional model of driven granular gas (already presented in [27]) are reported. In section III a one-dimensional and twodimensional version (in Boltzmann approximation) of the same model is discussed, reporting analogous results. Then, in sections [V] and $\mathrm{V}$, some theoretical interpretations are proposed in order to understand non-maxwellian behavior and try to relate it to the clusterization 
phenomenon. Section VI is devoted to conclusions and open problems.

\section{THE INELASTIC HARD-ROD ONE-DIMENSIONAL MODEL}

Such class of models originates from the seminal paper of $\mathrm{Du}, \mathrm{Li}$ and Kadanoff [16] who considered $N$ identical hard rods confined between a thermal and a reflecting wall. In this model one has a statistically steady state as the result of the balancing between the dissipation of the kinetic energy due to the collisions between the rods and the energy reinjection due to the thermal wall; the latter supplies energy only to the last particle, which in turn transfers energy and momentum to the rest of the system, producing a somehow trivial cluster near the opposite wall. Such a state represents a breakdown of the equipartition of the energy in a stationary non-equilibrium system; however, its existence comes about as an artifact, since it is due to the peculiarity of the boundary conditions. In fact, in the model introduced in ref. [16] the mean kinetic energy per particle,

$$
E=\frac{1}{2 N} \sum_{i=1}^{N}<v_{i}(t)^{2}>
$$

and the mean dissipated energy per particle per unit time,

$$
W=\frac{1}{\Delta t} \sum_{j}<(\Delta E)_{j}>,
$$

(where $(\Delta E)_{j}$ is the energy loss during the $j_{t h}$ collision occurred in the time interval $[t-$ $\Delta t / 2, t+\Delta t / 2]$, and $\langle>$ is the time average), decay exponentially with the number $N$ of particles, as shown in figure (1). Finally, within the Kadanoff model only a small region of parameter space can be explored: since $r$, the restitution coefficient, defined below, must satisfy the inequality $N(1-r)<1$ in order to avoid inelastic collapse [10], [12].

Williams et al. 18 proposed an alternative heating mechanism. The idea is to supply kinetic energy to every particle by means of a random acceleration at every time step. Since the dissipation due to inelastic collisions is not effective in balancing the increase of energy coming from the random kicks (the latter is independent from the velocities, while the former is proportional to them), the authors subtract the average velocity of the center of mass of the system from the velocity of each particle at every time step in order to avoid energy divergence and total non-conservation of the total momentum. Though this method is numerically efficient, it does not appear realistic from a physical point of view.

Hereafter, we propose a model [27] consisting of $N$ identical particles of mass 1 on a ring of length $L$. Between collisions, each particle obeys to the following Langevin equation [28]:

$$
\begin{gathered}
\frac{d v_{i}}{d t}=-\frac{v_{i}}{\tau}+\sqrt{\frac{2 T_{F}}{\tau}} f_{i}(t) \\
\frac{d x_{i}}{d t}=v_{i}(t)
\end{gathered}
$$


where $1 \leq i \leq N, \tau$ and $T_{F}$ are the relaxation time due to viscous effects and the thermal bath temperature respectively, $f_{i}(t)$ is a standard white noise with zero average and $<$ $f_{i}(t) f_{j}\left(t^{\prime}\right)>=\delta_{i j} \delta\left(t-t^{\prime}\right)$.

In addition to these equations, the particles mutually collide according to the following rules:

a) only binary collisions are considered,

b) each collision is instantaneous,

c) the post-collisional velocities are related to the pre-collisional ones by the equations:

$$
\begin{aligned}
& v_{i}^{\prime}=\frac{1-r}{2} v_{i}+\frac{1+r}{2} v_{j} \\
& v_{j}^{\prime}=\frac{1+r}{2} v_{i}+\frac{1-r}{2} v_{j}
\end{aligned}
$$

where $r$ is the restitution coefficient. In this way, the momentum is conserved in the collisions, while the kinetic energy of center of mass is rescaled by $r^{2}$, i.e.

$$
\left(v_{i}^{\prime}-v_{j}^{\prime}\right)^{2}=r^{2}\left(v_{i}-v_{j}\right)^{2}
$$

The elastic case is $r=1$, while for $r=0$ the colliding particles have no relative motion after the collision: they move together with the velocity of the center of mass. It has to be noted that, in one dimension, the size of the particles is not a significant parameter, because of the "hard" nature of collisions: the particles never deform (this effect is kept into account in the restitution coefficient picture) and therefore only the length of spacings between particles is important, that is $L$ (see [16])

In absence of collisions, each particle would perform a Brownian motion reaching, for $t>>$, a stationary state with a gaussian velocity distribution:

$$
P\left(v_{i}\right)=\frac{1}{\sqrt{2 \pi T_{F}}} \exp \left(-\frac{v_{i}^{2}}{2 T_{F}}\right)
$$

and a diffusive behavior:

$$
<\left(x_{i}(t)-x_{i}(0)\right)^{2}>=2 D t
$$

where $D=T_{F} \tau / 2$ is the diffusion coefficient. The interpretation of the model is straightforward: the environment supplies kinetic energy to the system as a thermal bath at temperature $T_{F}$. The viscous term (with characteristic time $\tau$ ) is naturally introduced to take into account different friction effects, such as contact friction with boundaries, particle-fluid interaction, tangential inter-particle friction and energy transfer among different degrees of freedom. Experimental fluidized beds, see for example [29], are an example of phenomena in which a viscous damping and a noisy term are naturally present. As noted before, in [23] is presented an experiment showing strong analogies with our model.

When collisions are considered, another characteristic time emerges, that is the average collision time $\tau_{c}$ between two successive encounters. An estimate of $\tau_{c}$, as a function of average density and typical velocity, is 


$$
\tau_{c} \sim \frac{L}{2 N \sqrt{<v^{2}>}}
$$

It is natural to assume that $\left\langle v^{2}>\right.$ reaches a stationary value with statistical fluctuations (of order $\sim 1 / N)$, as it is observed in simulations. In the following we shall refer to the quantity

$$
T_{g}=<v^{2}>=\lim _{\left(T-T_{0}\right) \rightarrow \infty} \frac{1}{\left(T-T_{0}\right) N} \sum_{i=1}^{N} \int_{T_{0}}^{T} v_{i}(t)^{2} d t
$$

as to the granular temperature of the system. Note that the system is not at equilibrium, therefore $T_{g}$ is not a temperature in a proper thermodynamic sense: it may be different, if one measures it at different scales or in different subsystems (as it will be shown later).

In all the simulations performed, we use $L / N=1$ and $T_{F}=1$ and the measured $T_{g} \leq T_{F}$ is almost always found greater than 0.1 (and never drops below 0.03). ¿From eq. (12) we can estimate $0.5<\tau_{c}<5$.

The presence of two time scales $\left(\tau\right.$ and $\tau_{c}$ ) leads to two different regimes. As $\tau_{c}$ varies in a small range (less than one order of magnitude), we could tune the parameter $\tau$ to observe these two phases:

A) When $\tau<<\tau_{c}$ the effect of collisions is rapidly overwhelmed by the Brownian motion (i.e. collisions are rare events) and the system behaves as a collection of weakly interacting random walkers or, equivalently, as an ideal gas in equilibrium at a temperature $T_{g}$ not far from the temperature $T_{F}$ of thermal bath (one can be convinced of this also looking to the Boltzmann equation of the system, reported in the next section).

B) In the opposite limit $\tau_{c}<<\tau$ the collisions dominate the dynamics and strongly compete against the driving mechanism. In this regime a statistically stationary state is still observed, in the sense that macroscopic averages are well defined, and interesting phenomena emerge:

a) a strongly inhomogeneous spatial distribution (clusterization)

b) a deviation of velocity distribution from gaussian behavior. These phenomena are more and more pronounced with decreasing values of the restitution coefficient $r$.

The simulations have been performed using a fixed step $\Delta t$ integration of eqs. (6) and (7) where $\Delta t<<\tau_{c}$ and an event driven check of collisions during every time step. For low values of $r$ we observed an exponential decreasing of collision time, much shorter than the integration time $\Delta t$. We discarded these simulations, interpreting them as examples of inelastic collapse [12]. The critical value of $r$, for the appearance of collapse, increases with $\tau$ : in the limit $\tau \rightarrow \infty$ the thermal bath disappears and the system becomes a granular cooling model with critical value, for inelastic collapse, estimated by $N(1-r) \sim 1$ as noted before.

In figure (2) we report $T_{g}$ and $W$ vs. the restitution coefficient $r$ for different $\tau$.

A simple relation between $T_{g}$ and $W$ may be obtained. The variation of the kinetic energy due to Langevin dynamics is 


$$
\begin{aligned}
(\delta E(t))_{\text {Lang }}= & \frac{1}{2 N} \sum_{i=1}^{N}\left(v_{i}(t)+\delta v_{i}(t)\right)^{2}-\frac{1}{2 N} \sum_{i=1}^{N} v_{i}^{2}(t)= \\
& =\frac{1}{2 N} \sum_{i=1}^{N}\left(\delta v_{i}(t)\right)^{2}+\frac{1}{2 N} \sum_{i=1}^{N} v_{i}(t) \delta v_{i}(t)
\end{aligned}
$$

where $\delta v_{i}$ is the velocity variation during a time interval $d t$ in equation (6), from which we obtain the relations:

$$
\begin{gathered}
\lim _{d t \rightarrow 0}\left\langle\frac{\left.\delta v_{i}(t)\right)^{2}}{d t}\right\rangle=\sqrt{\frac{2 T_{F}}{\tau}} \\
\lim _{d t \rightarrow 0}\left\langle\frac{v_{i}(t) \delta v_{i}(t)}{d t}\right\rangle=-\frac{\left\langle\left(v_{i}(t)\right)^{2}\right\rangle}{\tau}
\end{gathered}
$$

where the $\langle\ldots\rangle$ average is taken over different realizations of stochastic process $f_{i}(t)$. Recalling the definition of $T_{g}$, using equations (4), (5), and inserting eqs. (15) and (16) into eq. (14), and assuming the ergodicity, one obtains:

$$
W=\frac{T_{F}-T_{g}}{\tau}
$$

The numerical check of such relation is shown in figure (3)

Though the system is statistically stationary, the instantaneous density of particles is rapidly evolving. To get an idea of different density profiles in the two regimes (homogeneous and clusterized), look at fig.(田).

The density distribution becomes fractal in the clusterized regime, as can be verified measuring the correlation dimension $d_{2}$ [30] that we computed from the correlation function

$$
C(R)=\frac{1}{N^{2}\left(T-t_{0}\right)} \int_{t_{0}}^{T} d t \sum_{i, j} \theta\left(R-\left|x_{i}(t)-x_{j}(t)\right|\right) \sim R^{d_{2}}
$$

with $t_{0}$ the time after which one can assume the system is in a typical situation and $T$ is the duration of the simulation. $\mathrm{C}(\mathrm{R})$ is shown for homogeneous and clusterized situations in figure (5). In figure (6) is presented a summary of $d_{2}$ measurements as function of parameters $\tau$ and $r$.

The clusterization may quantitatively characterized by means of an entropy defined as

$$
h_{M}=-\sum_{j=1}^{M} \frac{m_{j}}{N} \log \frac{m_{j}}{N}
$$

where the ring of length $L$ is divided in $M$ equal boxes (i.e., segments) and $m_{j}$ is the number of particles in the $j_{t h}$ box. The entropy $h_{M}$ attains its maximum value $h_{M}=\log M$ when $m_{j}=N / M$ for every box $j . h_{M}$ decreases as the density distributions becomes more and more clusterized. For a non-clusterized (but fluctuating) density, we have a Poisson distribution for $m_{j}$, that is (with $\lambda=N / M$ )

$$
f\left(m_{j}\right)=\frac{\lambda^{m_{j}}}{m_{j} !} \exp (-\lambda)
$$


from which it can be (numerically) calculated the effective entropy for homogeneous regime $h_{M}^{*}$. In figure (7) are presented many measurements of $H_{M} / H_{M}^{*}$ where $H_{M}=\exp \left(<h_{M}>\right)$, $H_{M}^{*}=\exp \left(<h_{M}^{*}>\right)$ and $<>$ is the time average. The quantity $H_{M} / H_{M}^{*}$ basically gives an indication of the fraction of non-empty boxes in a typical snapshot.

In figure (8) is shown the distribution of velocities, obtained sampling the velocities of all particles for very long times, in the two different regimes (a quasi-equilibrium case with $\tau=0.01, r=0.99$ and an out of equilibrium case with $\tau=100, r=0.7)$. In the quasiequilibrium regime the distribution is very well fitted by a gaussian. As a general result, when $\tau>>\tau_{c}$ the velocity distribution ceases to be gaussian and the high velocity tails decay more slowly to zero. The deviation becomes more pronounced as the restitution coefficient $r$ decreases. In the figure a theoretical fit is also plotted for the non-gaussian distribution. This fit will be discussed below, in section एV.

In figure (9) the density distributions in the homogeneous and clusterized regimes are shown (respectively $\tau=0.01, r=0.99$ and $\tau=100, r=0.7$, with $N=300$ ), i.e. $f_{M}(m)$ where $m$ is the number of particles in a box when the ring is divided in $M$ boxes (this distribution, as all the others, is obtained sampling data for very long times). The homogeneous regime is very well fitted by a Poisson distribution, as noted in equation (20). The clusterized regime presents an exponential long tail and a power law for the low density boxes: the function $\frac{1}{m} e^{-c m}$ with $c=0.14$ fits very well the data and is consistent with the theoretical interpretation given in section $\sqrt{\mathrm{V}}$.

The figure (10) represents the box granular temperature $T_{M}(m)$ as a function of the number of particles $m$ in a box:

$$
T_{M}(m)=\frac{1}{m} \sum_{j=1}^{m}\left(v_{j}-<v>_{m}\right)^{2}
$$

where $\langle v\rangle_{m}$ is the average velocity (typically close to zero when $m>>1$ ) in the box and $M$ is the number of boxes on the ring of length $L$. The figure shows this function (averaged over very long times) for both the regimes: in the gaussian case (with $\tau=0.01$ and $r=0.99$ ) we observe that $T_{M}(m)$ is a constant, while in the non-gaussian case $(\tau=100, r=0.7)$ it is a power law, i.e. $T_{M}(m) \sim m^{-\beta}$ with $\beta=0.5$. The exponent $\beta$ depends on the values of $\tau$ and $r$.

\section{THE BOLTZMANN EQUATION APPROXIMATION}

A natural question now arises: can we expect that the above results are general and independent from the dimensionality of the system? Or are these an artifact of the one dimensional dynamics? An answer may come from the Boltzmann equation for the one particle distribution $P(x, v, t)$ [31]:

$$
\begin{array}{r}
\frac{\partial P}{\partial t}+\frac{\partial(v P)}{\partial x}-\frac{1}{\tau} \frac{\partial(v P)}{\partial v}-\frac{T_{F}}{\tau} \frac{\partial^{2} P}{\partial v^{2}}=\left.\frac{\partial P}{\partial t}\right|_{\text {coll }} \\
\left.\frac{\partial P}{\partial t}\right|_{\text {coll }}=\frac{4 \Lambda}{(1+r)^{2}} \int d v^{\prime}\left|v^{\prime}-v\right| P\left(x, v^{\prime}, t\right) P\left(x,\left(2 v-(1-r) v^{\prime}\right) /(1+r), t\right)- \\
-\Lambda \int d v^{\prime} P\left(x, v^{\prime}, t\right) P(x, v, t)\left|v^{\prime}-v\right|
\end{array}
$$


where $\Lambda \sim 1 / \tau_{c}$ is the mean collision rate per particle. In the limit of elastic collisions $(r=1)$ the collision integral (23) disappears and the stationary solution of eq. (22) is $P(x, v, t) \propto \exp \left(-v^{2} / 2 T_{F}\right)$. This is related to the fact that in the elastic limit a collision between two particles is nothing but a change of the labels of the two particles and therefore the collisions are not relevant at all.

The main approximation in eq.(22) is the Boltzmann Stosszahlansatz, according to which the correlation between two close particles is neglected and one writes:

$$
P_{2}\left(x, x^{\prime}, v, v^{\prime}, t\right)=P(x, v, t) P\left(x^{\prime}, v^{\prime}, t\right)
$$

As equation (22), as far as we know, cannot be solved analytically, we consider a stochastic process based on the Bird algorithm [32], whose statistical features are identical to those of the Boltzmann equation. $N$ particles move on a torus (in $d$ dimensions) of linear size $L$ (i.e., the area of the d-torus is $L^{d}$ ). The time is discretized in intervals of duration $\Delta t$. A collision time $\tau_{c}$ is fixed a priori: this means that two particles collide, during $\Delta t$, with a probability $p=\Delta t / \tau_{c}\left(\Delta t<<\tau_{c}\right.$, as usual).

At each discrete time $t_{k}=k \Delta t$, positions and velocities are upgraded according to eqs. (6) and (7). Then for each particle $i$ a random number $y$ is extracted out of a uniform distribution in the interval $[0,1]$ : if $y>p$ no collision occurs, otherwise the particle $i$ collides with a particle $j$ such that $\left|x_{i}\left(t_{k}\right)-x_{j}\left(t_{k}\right)\right|<l(l<<L)$, chosen with probability proportional to $\left|v_{i}\left(t_{k}\right)-v_{j}\left(t_{k}\right)\right|$. The collision rule, in one dimension, is the same as before and it is extended to the two and three dimensional cases in a natural way: after a collision $\mathbf{v}_{\mathbf{i}}{ }^{\prime}-\mathbf{v}_{\mathbf{j}}{ }^{\prime}=r \hat{\epsilon}\left(\mathbf{v}_{\mathbf{i}}-\mathbf{v}_{\mathbf{j}}\right)$ where $\hat{\epsilon}$ is a unit vector with random orientation.

It can be demonstrated [33] that for this process, in the limit $N \rightarrow \infty, p \rightarrow 0, l \rightarrow 0$, $\Delta t \rightarrow 0$, the evolution of the probability distribution $P(x, v, t)$ of the Bird process is governed by the Boltzmann equation (22).

In this model there are two parameters, $\tau_{c}$ and $l$. The first one was already an observable of the previous model (where it was almost constant). The second one, instead, represents the collision range and replaces the radius of the particles, so it is related, in some way, to the total volume fraction $\nu=k N / L^{d}$ with $k$ the volume of one particle, which is not explicitly considered in this model and in the previous one. Furthermore, we cannot expect to find a fractal scaling at a range lower than $l$, and, moreover, no inelastic collapse can be observed, as short range correlations are neglected. It should be noted, finally, that the imposed collision time $\tau_{c}$ is larger (but of the same order of magnitude) than the one really measured in the simulations, $\tau_{c}^{*}$, because a particle collides with probability $p=\Delta t / \tau_{c}$ only if there is another particle at distance lower than $l$. One can expect that $\tau_{c}^{*} \rightarrow \tau_{c}$ as the clusterization becomes more and more pronounced.

In all the simulations of this model we kept $T_{F}=1$ and $N / L^{d}=1$, we rarely changed $\tau_{c}$ and $l$ and we explored the space of parameters $\tau$ and $r$ as in the previous section. All the results previously obtained were recovered in this approximation with $1 \leq d \leq 3$, showing that they are general properties of a granular system subject to such a heating mechanism as that of eqs. (6), (7). A rapid overview of significant measurements, in one and two dimensions, follows.

We stress the fact that also in this model the system reaches a statistically stationary state after a transient, and also in this model there are two different regimes: the quasiequilibrium regime $\left(\tau<<\tau_{c}\right)$ and the out-of-equilibrium one $\left(\tau>>\tau_{c}\right)$. 
The clusterization phenomenon is shown in figures (111), (12) and (13) where the density snapshots and the correlation functions $C(R)$ (defined in eq. (18)) are presented. It is observed the predicted reduction of the fractal scale (more evident in the two-dimensional model) due to Boltzmann approximation.

The existence of a good thermodynamic limit is shown in the figures (14) and (15) where the $N$-dependence of some observables is plotted: we show the granular temperature $T_{g}$, the fractal dimension $d_{2}$ and the collision rate $\chi=1 / \tau_{c}^{*}$, all in the out-of-equilibrium regime.

The same quantities are plotted in figures (16) and (17) against the restitution coefficient $r$ in the same regime (these plots are analogous to those of figures (22) and (60)). Note that $\tau_{c}^{*}$ approaches $\tau_{c}$ when $r$ decreases, as it is expected. The diffusion coefficients, also plotted in those figures, will be discussed in the conclusion.

The distributions of velocities are presented in figure (18) and (19). The non-gaussian behavior in the second regime $\left(\tau>>\tau_{c}\right)$ is still clearly observed. In figure (20) a distribution of velocities restricted in the following way is presented: we sampled the velocities of one particle only when there were other $m(m=1$ and $m=5)$ particles in a box of radius $R$ $(l<<R<<L)$ centered on that particle. This is a sort of velocity distribution at fixed density. The plot shows a less pronounced deviation from the gaussian, in agreement with the statement (discussed in section $\mathbb{I V}$ ) that there is a local equilibrium with a temperature that depends upon the local density, in order to have a stationary distribution of clusters.

The analogue of the figure (9) and (10) are the figures (21), (22), (23) and (24). Again the density distribution $f_{M}(m)$ is a Poisson function when the system is not clusterized and a function of the form $\exp (-\alpha m) / m^{\beta}$ in presence of clustering. The box granular temperature $T_{M}(m)$ still presents a constant behavior (as expected) at equilibrium and a power law $m^{-\gamma}$ in the non-gaussian regime. It does not seem possible to easily find a relation between $\alpha, \beta$, $\gamma$ and the other observables (as $d_{2}$ or $T_{g}$ ).

In summary, the exact model is perfectly reproduced in this Boltzmann approximation, at large $N$, not only confirming the existence of a thermodynamic limit, but also showing that this system may be studied analytically in order to better understand this kind of driven granular kinetics. In the following sections some theoretical interpretations will be discussed.

\section{A MODEL TO EXPLAIN CLUSTERIZATION}

In this section we address the problem of the microscopic origin of the clusterization. In order to do that, we study a class of models in which the system is composed by $M$ boxes and $N$ particles in a mean-field model, assuming that the boxes have infinite connectivity. One starts with a certain configuration and let the system evolve with an exchange dynamics in which, at each time step, one particle moves from one box to another, both boxes being chosen randomly. The probability for each single exchange is model-dependent and it will be our tuning-parameter to scan the different phenomenologies. Our goal is to understand in a quantitative way how the microscopic dynamics affects the clustering properties of the system. In particular we shall try to recover the results, obtained in the framework of the models previously introduced, for the density distributions in the clusterized and homogeneous cases (see Figs. (91), (21). (22)). 
The models are defined in terms of master equations for the probability $P_{m}$ of having a box with $m$ particles, assigning transition rates for landing in a box with $m$ particles $W_{i n}(m)$ and for leaving a box with $m$ particles $W_{\text {out }}(m)$. It must be

$$
W_{\text {in }}(N)=W_{\text {out }}(0)=0
$$

and the normalization conditions must be satisfied:

$$
\sum_{m=0}^{\infty} P_{m}=1 \quad \sum_{m=0}^{\infty} m P_{m}=\frac{N}{M}=\lambda \quad \sum_{m=0}^{\infty} W_{i n}(m) M P_{m}=1
$$

The general question is: what is the asymptotic stationary distribution for the average number of boxes with $m$ particles, $P(m)$ ?

The simplest case we can consider is the one in which each single movement is independent of the state of the departing and of the landing box. In this case there is no bias in the movements and $W_{\text {in }}(m)$ and $W_{\text {out }}(m)$ do not depend upon $m$ :

$$
W_{\text {in }}(m)=W_{\text {out }}(m)=\frac{1}{M}
$$

and the general master equation reads

$$
\begin{aligned}
M^{2} \frac{d P_{m}}{d t}= & P_{m-1}\left(P_{m-1}-\frac{1}{M}\right)+2 P_{m+1} P_{m-1}+P_{m+1}\left(P_{m+1}-\frac{1}{M}\right)+ \\
& +P_{m+1}\left(1-P_{m}-P_{m+1}-P_{m-1}\right)+\left(1-P_{m}-P_{m-1}-P_{m+1}-P_{0}\right) P_{m-1}+ \\
& -P_{m-1} P_{m}-2 P_{m}\left(P_{m}-\frac{1}{M}\right)-P_{m} P_{m+1}-P_{m}\left(1-P_{m-1}-P_{m}-P_{m+1}\right)+ \\
& -\left(1-P_{m}-P_{m+1}-P_{m-1}-P_{0}\right) P_{m} \\
M^{2} \frac{d P_{0}}{d t}= & P_{1}\left(1-P_{0}-\frac{1}{M}\right)-\left(1-P_{1}-P_{0}\right) P_{0} \\
M^{2} \frac{d P_{N}}{d t}= & P_{1} P_{N-1}-P_{N}\left(1-\frac{1}{M}\right)
\end{aligned}
$$

In the limit of $M>>1$ one can neglect the $\frac{1}{M}$ terms in the right hand side of eq. (28) and easily get the stationary solution $\left(\frac{d P_{m}}{d t}=0\right)$

$$
P_{m}=A e^{-c m}
$$

with $A=1-e^{-c}$ corresponding to the normalization condition $\sum_{0}^{\infty} P_{m}=1$ and where $c$ is a constant depending on $N$ and $M: c=\ln \left(1+\frac{1}{\lambda}\right)$ with $\lambda=\frac{N}{M}$.

This result has to be compared with the probability $f_{M}(m)$ in the non-clusterized case of the previous sections. In order to do this it is necessary to recall that this result has been obtained with a small value of the number of boxes $M$. This means that one is very far from the limit $M>>1$ and this situation corresponds to a sort of coarse graining in the system in which each box (big box) is actually composed by a certain number of small boxes (whose number is such that $M>>1$ ). The problem can thus be formulated in the following way: given a system of $N$ particles distributed in $M_{\text {small }}$ boxes with the distribution $P_{m}$ given by eq. (30), what is the distribution $P_{m}^{*}$ for the particles in a system of $M_{b i g}$ boxes each one 
composed by $R\left(R=M_{\text {small }} / M_{\text {big }}\right)$ small boxes? The resulting distribution is easily written as

$$
P_{m}^{*}=\sum^{*} \prod_{i=1}^{R} P_{m_{i}}=A^{R} e^{-c m} F(m, R)
$$

where $\sum^{*}$ indicates the sum on the $\left\{m_{1}, \ldots, m_{R}\right\}$ such that $\sum_{i=1}^{R} m_{i}=m, F(m, R)$ is the number of ways of distributing $m$ particles in $R$ boxes and it is given by [34]:

$$
F(m, R)=\left(\begin{array}{c}
m+R-1 \\
m
\end{array}\right)
$$

With the help of (32) and using the Stirling formula, the expression (31) becomes (for $R>>N>>1$ )

$$
\begin{aligned}
P_{m}^{*}= & A^{R} e^{-c m} \frac{(m+R-1) !}{m !(R-1) !} \approx A^{R} e^{-c m} \frac{(m+R-1)^{m}(m+R-1)^{R-1}}{m !(R-1)^{R-1}} \approx \\
& \approx A^{R} e^{-c m} \frac{R^{m}}{m !}=\frac{A^{R}}{m !} e^{-m\left(\ln \left(1+\frac{M_{\text {small }}}{N}\right)-\ln \frac{M_{\text {small }}}{N}-\ln \frac{N}{M_{\text {big }}}\right)} \approx \\
& \approx e^{-\lambda^{*}} \frac{\left(\lambda^{*}\right)^{m}}{m !}
\end{aligned}
$$

It has been used the definition of $R$, the fact that $c=\ln \left(1+M_{\text {small }} / N\right)$ and that $M_{\text {small }} / N>>$ 1. In the last passage $\lambda^{*}=N / M_{\text {big }}$ has been introduced and $A^{R}$ has become $e^{-\lambda^{*}}$, as can be verified when $\lambda^{-1}=M_{\text {small }} / N>>1$. It has been shown, therefore, that the coarse grained version of the solution of (28) is exactly the Poisson distribution found in the simulations, in the non-clusterized regime (see figs. (9), (21) and (22)).

Let us consider now one case where the transition rates for the particle jumps depend on the contents of the departing and landing boxes. This corresponds to impose some sort of bias to the system that could well reproduce the situation one has in the clusterized cases due to the inelasticity. We consider in particular the following case, defined by the transition rates:

$$
\begin{aligned}
W_{\text {in }}(0) & =\frac{1}{M} & & \\
W_{\text {in }}(m) & =\left(1-P_{0}\right) \frac{m}{N} & & \text { for } 0<m<N \\
W_{\text {out }}(m) & =\frac{m}{N} & & \text { for } 0<m \leq N
\end{aligned}
$$

These transition rates, that satisfy the relations (26), have the following interpretation. The probability to land on a box containing already $m$ particles is proportional to the number of particles because this mimics the inelastic collision with a cluster of $m$ particles. On the other hand the departure from a box containing already $m$ particles has a probability proportional to $m$ because the probability to select one particle in that particular box is proportional to $m$.

Neglecting as usual the terms of the order of $\frac{1}{M}$, and after simplifications, the stationary master equations write: 


$$
\begin{array}{r}
P_{m+1}(m+1)+\left(1-P_{0}\right)(m-1) P_{m-1}-P_{0} m P_{m}=0 \\
P_{1}-\frac{N}{M} P_{0}=0 \\
\frac{P_{1}}{N} P_{N-1}\left(1-P_{0}\right) \frac{N-1}{N}-P_{N}\left(1-\frac{1}{M}\right) \frac{1}{M}=0
\end{array}
$$

The solution in this case is given by:

$$
P_{m}=A \frac{1}{m} e^{-\alpha m} \quad P_{0}=1-e^{-\alpha}
$$

with $A=\lambda\left(e^{\alpha}-1\right)$ and $\lambda=\frac{N}{M}$. $A$ and $\alpha$ are related by an implicit equation obtained imposing the condition $\sum_{0}^{N} P_{m}=1$, that in the limit $N \rightarrow \infty$ becomes

$$
1-e^{-\alpha}-A * \ln \left(1-e^{-\alpha}\right)=1
$$

In the clusterized case we expect the solution to be self-similar, in the sense that $P_{m}$ has the same behavior of $P_{m}^{*}$, and the coarse graining previously performed should not change the solution (36), apart a rescaling of $\lambda$ and $\alpha$.

It must be noted that, as $A$ must be finite, when $N \rightarrow \infty$ (and $M$ is fixed) $\alpha$ has to go to zero, while $\alpha$ diverges when $N / M$ goes to zero. It is natural to think to $\alpha$ as to the inverse of the characteristic "mass" of a cluster, that is the typical number of particles in it. In this sense the term $\exp (-\alpha m)$ acts as a finite-size cut-off for the self-similar distribution $P_{m} \sim 1 / m$.

The solution (36) is in excellent agreement with the numerical results obtained in the previous sections. In particular in the case $N=300 M=100$ of the one-dimensional model of Sect.II one recovers the density distribution with the correct value of $\alpha \simeq 0.14$ (see fig. (9)) .

To get the other observed behaviors of density distribution $P_{m} \sim e^{-\alpha m} / m^{\beta}$ (see figs. (21) and (22)), it is enough to change the transition rates appearing in eqs. (34) into the following:

$$
\begin{aligned}
W_{\text {in }}(0) & =\frac{1}{M} & & \\
W_{\text {in }}(m) & =\mu\left(1-P_{0}\right) m^{\beta} & & \text { for } 0<m<N \\
W_{\text {out }}(m) & =\mu m^{\beta} & & \text { for } 0<m \leq N
\end{aligned}
$$

where $\mu$ is a normalizing constant:

$$
\mu=\left(M \sum_{i}^{N} P_{m} m^{\beta}\right)^{-1} .
$$

Now, we can go a step further relating the clustering properties of the system to the velocity distribution. In order to do that we consider the following quantities: the distribution of boxes, $f_{M}(m)$, containing a given number $m$ of particles and the velocity variance $T_{M}(m)$, in a box occupied by $m$ particles. We consider first the non-clusterized case $\left(\tau<<\tau_{c}\right.$ and $r \simeq 1$ ). Within this regime we find from the simulations that: 


$$
\begin{aligned}
T_{m}^{\text {elas }}(m) & \simeq \text { const } . \\
f_{M}^{\text {elas }}(m) & =\frac{\lambda^{m} e^{-m}}{m !} .
\end{aligned}
$$

By assuming in each box a gaussian velocity distribution with a constant variance $T_{M}^{\text {elas }}(m)$ it turns out that the global velocity distribution $P^{\text {elas }}(v)$ is gaussian. Let us recall that the Poisson distribution is the one associated with a process of putting independently $\lambda N$ particles into $N$ boxes.

Let us turn to the non-elastic case. If $\tau=100$ and $r=0.7$, considering the occupied boxes $(m>0)$, we obtain from the simulations the following relations

$$
\begin{aligned}
T_{M}^{i n e l}(m) & \sim m^{-\beta} \\
f_{M}^{i n e l}(m) & =\frac{e^{-\alpha m}}{m},
\end{aligned}
$$

with $\beta \simeq 0.5$ and $\alpha \simeq 0.14$. Let us compute from these scalings the global velocity distribution. Taking into account that the spatial probability distribution of the particles is $f_{M}(m)$ and assuming that their local velocity distribution is gaussian, but with a variance $T_{M}(m) \simeq m^{-\beta}$ which depends on the occupancy, we obtain, for the global velocity distribution $P_{\text {inel }}(v)$, and in the continuum limit:

$$
P_{\text {inel }}(v) \simeq \sum_{m=1}^{\infty} e^{\left(-\frac{v^{2} m^{\beta}}{2}\right)} e^{-\alpha m}
$$

We stress how the the distributions measured in the simulations are in very good agreement (see the dashed line in fig. (8)) with the numerical computation of eq. (46), which, in summary, has been obtained under only the following hypothesis:

(i) non-Poissonian distribution for the box occupancy $f_{M}(m) \propto e^{-\alpha m} / m$;

(ii) gaussian distribution of velocities in each box with a density-dependent variance $T_{M}(m) \propto m^{-\beta}$.

The hypothesis about the scaling relation between the velocity variance (i.e. $T_{M}(m)$ ) and the local density, apart from being justified numerically, can be understood in the following way. The stationarity and the scale-invariance of the cluster distribution, implies a certain distribution of lifetimes for the clusters. In particular each cluster has a lifetime which is inversely proportional to its size. The scale-invariant cluster-size distribution thus implies a scale-invariant distribution for the lifetimes. The cluster lifetime is strictly related to the variance of the velocity distribution inside the cluster itself. In order to ensure the stability of a cluster in a stationary state we have to require that the velocities of the particles belonging to it are not too different, or equivalently that the variance of the distribution is smaller the higher the density. So, given a scale-invariant distribution of clusters one would expect a scale-invariant distribution of variances, that is $T_{M}(m) \sim m^{-\beta}$.

In the next section the non-gaussian distribution of velocity will be related to clusterization with the help of a mean field model of driven granular gas. 


\section{A MODEL FOR THE CLUSTERING AND THE NON-GAUSSIAN BEHAVIOR}

In order to shed some light on the relationship between the spatial clusterization and the anomalous velocity distribution observed above, we present a simple theoretical model. For sake of notation simplicity, we discuss only the $1 \mathrm{~d}$ case. Let us treat the collisions in a mean-field like fashion and modify the Langevin dynamics plus collision rules by the following set of coupled equations for the velocities:

$$
\frac{d v_{i}}{d t}=-\frac{v_{i}}{\tau}+\frac{1}{N} \sum_{j=1}^{N} g\left(v_{i}-v_{j}\right)+\sqrt{\frac{2 T_{F}}{\tau}} f_{i}(t)
$$

where the second term in the r.h.s. determines the velocity change of the particle $i$ due to the collisions with the remaining particles and is chosen to mimick the inelastic behavior. This requirement poses some constraints about the form of the function $g\left(v-v^{\prime}\right)$ :

- The momentum conservation dictates the antisymmetric property, $g\left(v-v^{\prime}\right)=-g\left(v^{\prime}-\right.$ $v)$

- The inelasticity of the collision process requires $g\left(v-v^{\prime}\right)\left(v-v^{\prime}\right) \leq 0$

The Fokker-Planck equation of (47) is

$$
\begin{array}{r}
\partial_{t} P_{N}\left(v_{1}, \ldots, v_{N}, t\right)-\frac{1}{\tau} \sum_{i=1}^{N} \frac{\partial}{\partial v_{i}}\left(v_{i} P_{N}\left(v_{i}, \ldots, v_{N}, t\right)-\frac{T_{F}}{\tau} \sum_{i=1}^{N} \frac{\partial^{2}}{\partial v_{i}^{2}} P_{N}\left(v_{1}, \ldots, v_{N}, t\right)+\right. \\
+\sum_{i=1}^{N} \frac{\partial}{\partial v_{i}}\left[\frac{1}{N} \sum_{j=1}^{N} g\left(v_{i}-v_{j}\right) P_{N}\left(v_{1}, \ldots, v_{N}, t\right)\right]=0
\end{array}
$$

¿From the above equation, using the fact that in the limit $N \rightarrow \infty$ the mean field approximation holds, one can obtain an evolution equation for the 1-body velocity probability distribution which reads:

$$
\frac{\partial P(v, t)}{\partial t}-\frac{1}{\tau} \frac{\partial(v P(v, t))}{\partial v}-\frac{T_{F}}{\tau} \frac{\partial^{2} P(v, t)}{\partial v^{2}}+\frac{\partial}{\partial v} \int d v^{\prime} P(v, t) P\left(v^{\prime}, t\right) g\left(v-v^{\prime}\right)=0
$$

i.e. a sort of self consistent Boltzmann equation. ¿From eq. (49) one observes that the quantity:

$$
\int d v^{\prime} P\left(v^{\prime}, t\right) g\left(v-v^{\prime}\right)=G(v)=-\frac{\partial U(v)}{\partial v}
$$

which is a function of $v$ and a functional of $P(v)$, can be considered as an effective force acting on the particle generated by an effective potential $U$. Integrating once with respect to the velocity the stationary version of eq. (49) one can obtain the following equation:

$$
\left(-\frac{v}{\tau}+\frac{T_{F}}{\tau} \frac{\partial}{\partial v}+G(v)\right) P(v)=0
$$


The solution of eq. (51) is:

$$
P(v) \propto \exp \left(-\frac{\tau}{T_{F}}\left(\frac{v^{2}}{2 \tau}+U(v)\right)\right)
$$

In order to make some progress we consider the qualitative shape of $g\left(v_{i}-v_{j}\right)$. In eq. (47) the effect of collisions between the particles $i$ and $j$ in the unit of time is given by:

$$
\left.\frac{d}{d t}\left(v_{i}-v_{j}\right)\right|_{\text {coll }}=\frac{2}{N} g\left(v_{i}-v_{j}\right)
$$

The variation of momentum in an interval $d t$ can be rewritten as

$$
\left.\delta\left(v_{i}-v_{j}\right)\right|_{\text {coll }}=\delta q_{i j} \cdot\left(v_{i}-v_{j}\right)
$$

where $\delta q_{i j}$ is the analogue of $q=1-r$ in the model discussed in sections प1 and [II]. The important difference is that here $\delta q_{i j}$ represents the effect of all the collisions during $d t$, and thus can be associated to an effective restitution coefficient. Eq. (54) may be rewritten as:

$$
\left.\frac{d}{d t}\left(v_{i}-v_{j}\right)\right|_{\text {coll }}=\chi_{i j} q \cdot\left(v_{i}-v_{j}\right)
$$

where $\chi_{i j}$ is the number of collisions between the $i-t h$ and the $j-t h$ particles in the unit of time. Upon comparing eqns. (53) and (55) one obtains an expression for $g\left(v_{i}-v_{j}\right)$ :

$$
g\left(v_{i}-v_{j}\right)=\frac{2 \chi_{i j} q}{N}\left(v_{i}-v_{j}\right)
$$

Now it is easy to understand that $\chi_{i j}$ is a decreasing function of $\left|v_{i}-v_{j}\right|$ : indeed, a great number of collisions occurs when the pair $i, j$ belongs to a cluster (where $\left|v_{i}-v_{j}\right|$ is small), whereas the two particles rarely collide when they are out of a cluster (and $\left|v_{i}-v_{j}\right|$ is high). We can, therefore, make a rough estimate of $g\left(v_{i}-v_{j}\right)$, that is:

$$
\begin{array}{rr}
\left|g\left(v_{i}-v_{j}\right)\right| \sim \frac{\left|v_{i}-v_{j}\right|}{\tau_{c}} & \text { inside clusters } \\
\left|g\left(v_{i}-v_{j}\right)\right| \sim\left|v_{i}-v_{j}\right|^{\beta^{\prime}} & \text { outside clusters }
\end{array}
$$

where $\beta^{\prime}<1$. ¿From eq. (50) it appears that $\left.G(v) \sim g\left(v-v^{\prime}\right)\right|_{v^{\prime}=0}$ as the integration has to be performed with respect to the measure $P\left(v^{\prime}, t\right) d v^{\prime}$ that is strongly peaked at $v^{\prime}=0$. Finally, one can conclude from the same eq. (50) that

$$
\begin{array}{rlrl}
U(v) & \sim \frac{v^{2}}{\tau_{c}} & & v \lesssim \sqrt{T_{g}} \\
U(v) \sim v^{\beta} & v \gtrsim \sqrt{T_{g}}
\end{array}
$$

where $\beta=\beta^{\prime}+1<2$. It is clear now, looking at eq. (52), that when $\tau<\tau_{c}$ (i.e., in the non-clusterized regime) the argument of the exponential is dominated by $v^{2} / \tau$ and therefore a gaussian is expected for $P(v)$ with variance $T_{F}$. In the opposite regime, when $\tau>\tau_{c}$ the distribution is a gaussian with variance $\frac{\tau_{c}}{\tau} T_{F}$ at low velocities, a simple exponential (if $\beta=1$ ) 
at high velocities, and a gaussian with variance $T_{F}$ at extremely high velocities, but this very far tails practically cannot be observable. In figure (25) the tails of the distributions of velocities (from the simulation of the model of section III) for three different choices of parameters are presented: in case (a), when $\tau<\tau_{c}$, we observe a Gaussian distribution; in case (b), when $\tau>\tau_{c}$, we can fit the tail with the function $\exp \left(-v^{3 / 2} / b\right)$, and this is in agreement with the analytical calculation performed by van Noije and Ernst [22]; finally in case (c), when $\tau>>\tau_{c}$ we observe a simple exponential tail, as we may expect from the argument presented above.

\section{CONCLUSIONS AND OPEN PROBLEMS}

In this paper a class of models of granular gases in one and two dimensions has been studied by mean of computer simulations and analytical investigations. We think to this class of models as the natural, and more physical, extension of previous models in the domain of granular kinetics [16 - 20]. In the models here proposed, by effect of balance between Brownian driving and inelastic collisions, one has a good thermodynamic limit; furthermore, these models present a rich phenomenology as several regimes are observed by tuning the physical parameters, that is the time of viscous interaction $\tau$ and the coefficient of restitution for inelastic collisions $r$. The two extreme behaviors of those models are the Gaussian/homogeneous regime and the non-Gaussian/clusterized one. In the homogeneous phase, the system may be described almost as a perfect gas in equilibrium at a temperature close to that of the external driving (or a bit lower), showing the absence of densities instabilities and a Maxwellian distribution of velocities. The out-of-equilibrium phase, on the other side, presents strong fluctuations of density (clusters and collapse) with self-similar density distribution and a stationary fractal dimension, while there is a strong enhancement of high energy tails in the distribution of velocities. This dramatic breaking of the equipartition law has to be kept into account in modeling the hydrodynamics of granular media. Furthermore, we explained the origin of the different degrees of clusterization by means of a class of ballsin-the-boxes models, showing that the effect of inelasticity may be viewed as a bias to the transition rates of these random processes: in this context we showed that the non-Gaussian distribution of velocities is recovered assuming a local equilibrium with a temperature which depends on the local density. The non-Gaussian behavior has been also analytically investigated with the help of a model in which the effect of collisions is treated as a mean-field force on each particle and using the fact that this force has a different dependence on the impact velocity whether the particle is in a cluster or outside of it. Diffusion of particles has been also investigated in the simulation of models of section III: no anomalous diffusion has been observed. The diffusion coefficients for the non-Gaussian regime have been reported in figures (16) and (17) showing a weak (and apparently non-monotonic) dependency on the restitution coefficient $r$. A measure of velocity correlation function $\langle v(t) v(t+\tau)\rangle$, which appeared not to be a trivial exponential but likely a superposition of different exponentials (therefore still integrable in time), has convinced us that, even in the clusterized regime, the particles forget their previous velocities rather quickly due to collisions, that is: they enter and exit a cluster frequently enough to not affect average diffusion; however, in the clusterized regime the diffusion process is dominated by inter-particles collisions, whereas in the homogeneous one the diffusion is dominated by the Brownian motion imposed by the 
model. This is only a rough picture, to be furtherly investigated.

An important task to accomplish should be the research of an equation of state for this class of gases, useful in an eventual hydrodynamic description of them. The observed relation between local temperature and density (see figures (10), (23) and (24) and the discussion in section [IV]) is the starting point in this project. Analytical expressions of the pressure have to include the usual streaming term $\rho<v^{2}>$ (where $\rho$ is the local density and $\langle>$ is an ensemble average) as well as a collisional term which is important in the regions where the density is high: the streaming term, as a consequence of the scaling $\left\langle v^{2}>\sim \rho^{-\beta}\right.$, see the first of eqs. (45), is expected to be proportional to $\sim \rho^{1-\beta}$ if the picture of local Gaussian equilibrium is confirmed [35].

Acknowledgments We thank A. Petri, E. Caglioti and M. Marsili for useful discussions. V.L. acknowledges financial support under project ERBFMBICT961220 and FMRXCT980183. 


\section{REFERENCES}

[1] H.M. Jaeger and S.R. Nagel, Science 255, 1523 (1992); H.M. Jaeger, S.R. Nagel and R.P. Behringer, Phys. Today April 1996; H.M. Jaeger, S.R. Nagel and R.P. Behringer, Rev. Mod. Phys. 68, 1259 (1996) and references therein.

[2] C.S. Campbell, Annu.Rev.Fluid Mech. 22, 57 (1990).

[3] S.R. de Groot, P. Mazur, "Non-equilibrium thermodynamics", Dover Publications, (1984).

[4] R.A. Bagnold, Proc. R. Soc. London Ser. A 225, 49 (1954).

[5] P.K. Haff, J.Fluid.Mech. 134, 401 (1983).

[6] S. Chapman, T.G. Cowling, "The mathematical theory of non-uniform gases", Cambridge Univ. Press (1970, 3rd ed.).

[7] C.K.K. Lun, S.B. Savage, D.J. Jeffrey, N.Chepurniy, J. Fluid Mech. 140, 223 (1984).

[8] J.T. Jenkins, M.W. Richman, Phys.Fluids 28, 3485 (1985).

[9] S.E. Esipov, T. Pöschel, J.Stat.Phys. 86, 1385 (1997).

[10] I. Goldhirsch and G. Zanetti, Phys. Rev. Lett. 70, 1619 (1993).

[11] S. McNamara and W. R. Young, Phys. Fluids A 4, 496 (1992).

[12] S. McNamara and W. R. Young Phys.Fluids A 5, 34 (1993).

[13] S. McNamara and W. R. Young Phys.Rev. E 50, R28 (1994).

[14] S. McNamara and W. R. Young Phys.Rev. E 53, 5089 (1996).

[15] S. Luding, M. Huthmann, S. McNamara, A. Zippelius, cond-mat/9804003 (1998).

[16] Y. Du, H. Li, L. P. Kadanoff, Phys. Rev. Lett. 74, 1268 (1995).

[17] E. L. Grossman and E. Roman, Phys. Fluids 8, 3218 (1996).

[18] D.R.M. Williams, F.C. MacKintosh, Phys. Rev. E. 54, R9 (1996).

[19] M.R. Swift, M. Boamfa, S.J. Cornell, A. Maritan, Phys.Rev.Lett 80, 4410 (1998).

[20] G. Peng, T. Ohta, cond-mat/9710119 (1997).

[21] S.E. Esipov and T. Pöschel, J.Stat.Phys. 86, 1385 (1997).

[22] T.P.C. van Noije and M.H. Ernst, cond-mat/9803042 (1998) and references therein.

[23] J.S. Olafsen and J.S. Urbach, cond-mat/9807148.

[24] N.V. Brilliantov, F. Spahn, J. Hertzsch, T. Pöschel, Phys.Rev. E 53, 5382 (1996); N.V. Brilliantov and T. Pöschel, cond-mat/9803387.

[25] S. McNamara and S. Luding, cond-mat/9805250 and cond-mat/9805257.

[26] K.Geisshirt, P.Padilla, E.Præstgaard, S.Toxvaerd, Phys. Rev. E 57, 1929 (1998).

[27] A.Puglisi, V.Loreto, U.Marini Bettolo Marconi, A.Petri and A.Vulpiani, Phys. Rev. Lett. xx, xxx (1998) and A. Puglisi, Tesi di Laurea, Univ. of Rome La Sapienza (1997).

[28] S.Chandrasekhar, Rev. Mod. Phys 15, 1 (1948).

[29] G. M. Homsy, R. Jackson, and J. R. Grace, J. Fluid Mech. 236, 477 (1992); M. Ishida, T. Shirai and A. Nishiwaki, Powder Technol. 27, 1 (1980).

[30] P.Grassberger, I.Procaccia, Phys. Rev. Lett 50. 346 (1983).

[31] H.J.Kreuzer, Nonequilibrium Thermodynamics and its Statistical Foundations (Clarendon Press, Oxford), cap.7 (1981).

[32] G.A. Bird, Phys. Fluids 13, 2676 (1970).

[33] W. Wagner, J. Stat. Phys. 66, 1011 (1992) and C. Cercignani, R. Illner, M. Pulvirenti, "The Mathematical Theory of Dilute Gases", Springer Verlag (1994).

[34] We are indebted with Davide Cassi that recalled us the solution of this combinatorial problem. 
[35] D. Benedetto, E. Caglioti, J. A. Carrillo and M. Pulvirenti, J. of Stat. Phys. 91, 5/6 (1998), D. Benedetto, E. Caglioti and M. Pulvirenti, Math. Mod. Num. Anal. 31,5, p.615-641 (1997). D. Benedetto, E. Caglioti, F. Golse and M. Pulvirenti, A Hydrodynamic Model Arising in the Context of Granular Media preprint (1998). 


\section{FIGURES}
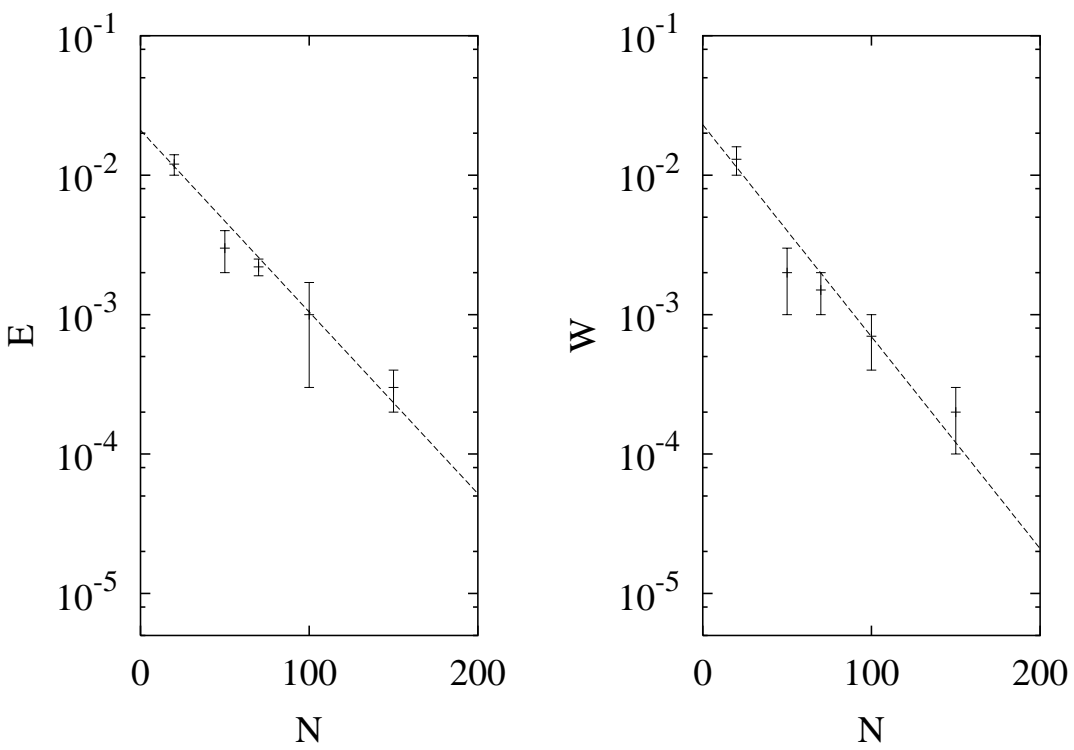

FIG. 1. Kinetic energy and dissipated energy per particle (as defined in equations (画) and (5)) vs. N, in Du et al. [16] model. Particle density $N / L=100$ is kept constants and $r=0.99$.
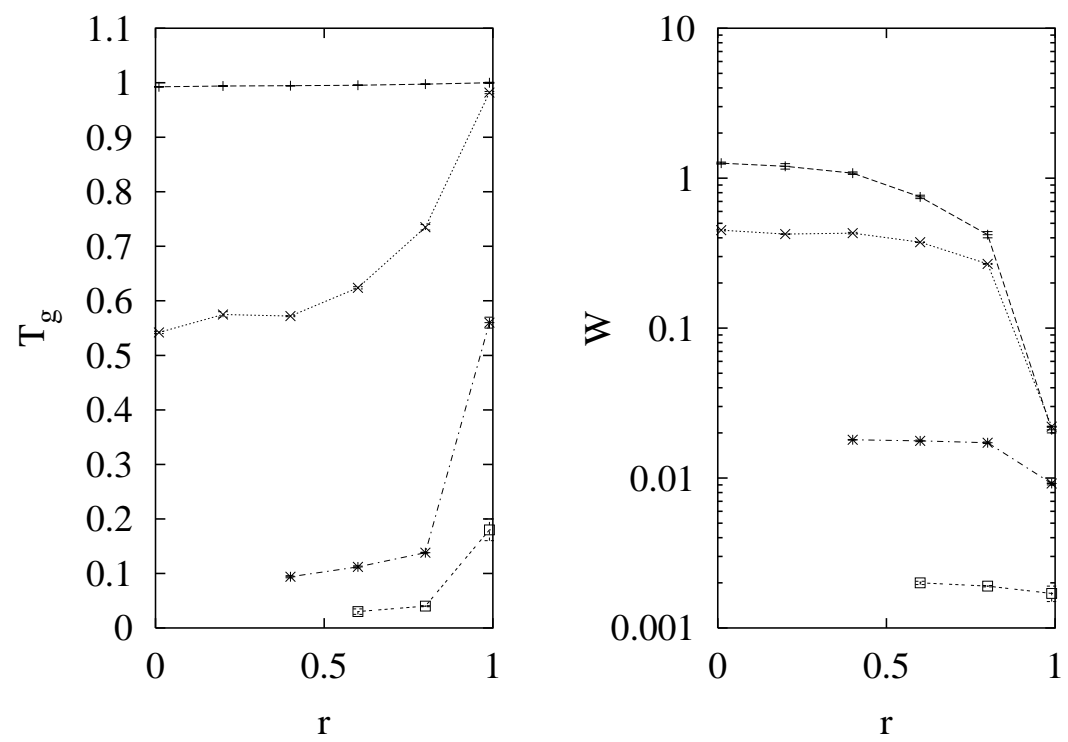

FIG. 2. The average granular temperature $T_{g}$ and the average dissipated energy per particle $W$ vs the restitution coefficient $r$ for different values of $\tau$ and $N=200$. From top to bottom: $\tau=0.01, \tau=2, \tau=100, \tau=1000$. 

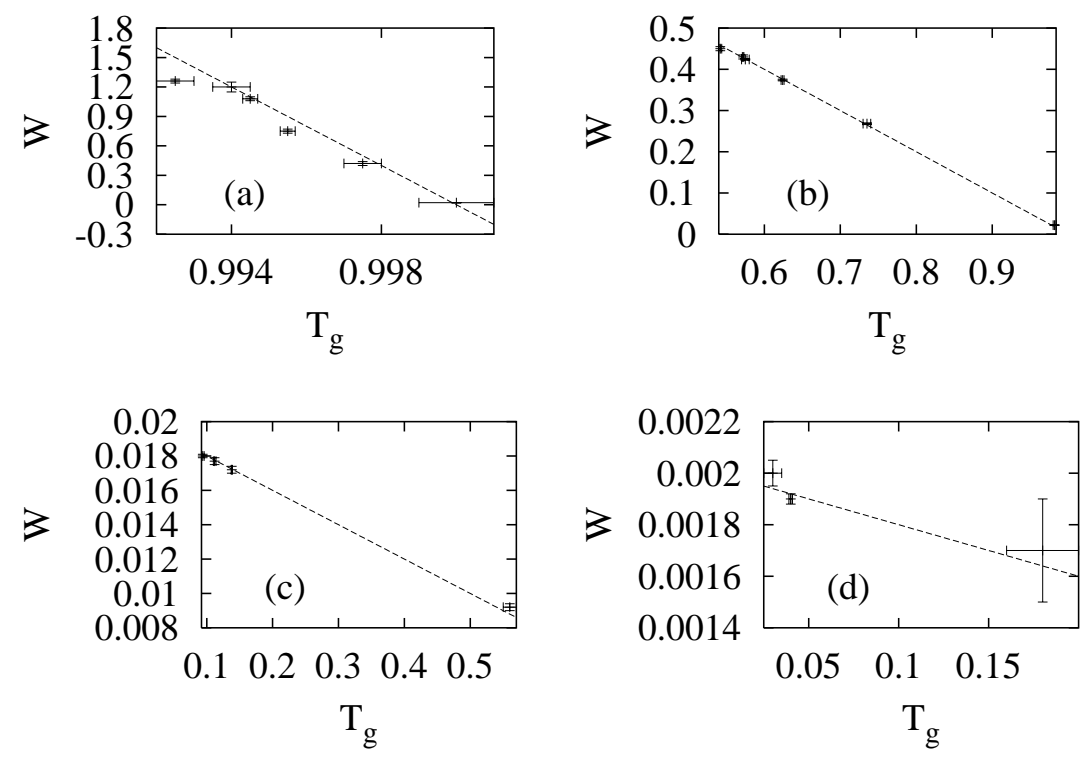

FIG. 3. Dissipated energy per particle $W$ versus the granular temperature $T_{g}$, for different $\tau$ and different $r: \tau=0.01$ (a), $\tau=2$ (b), $\tau=100$ (c), $\tau=1000$ (d). The dashed lines represent the relation (17)
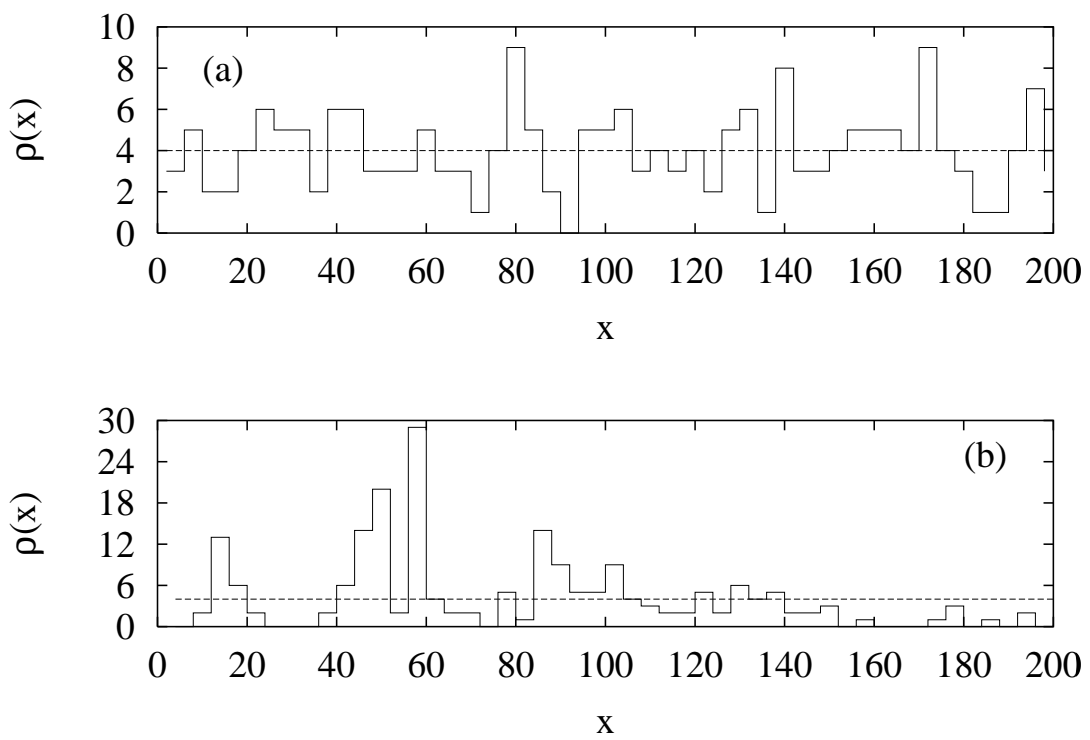

FIG. 4. Instantaneous density profiles $\rho(x)$ in two regimes: (a) quasi-equilibrium regime $(\tau=0.01, r=0.99)$ and (b) non-equilibrium regime with clusters $(\tau=100, r=0.6)$. In both histograms $N=200$ and the dashed horizontal lines represent the average density, equal to four particles per bin 


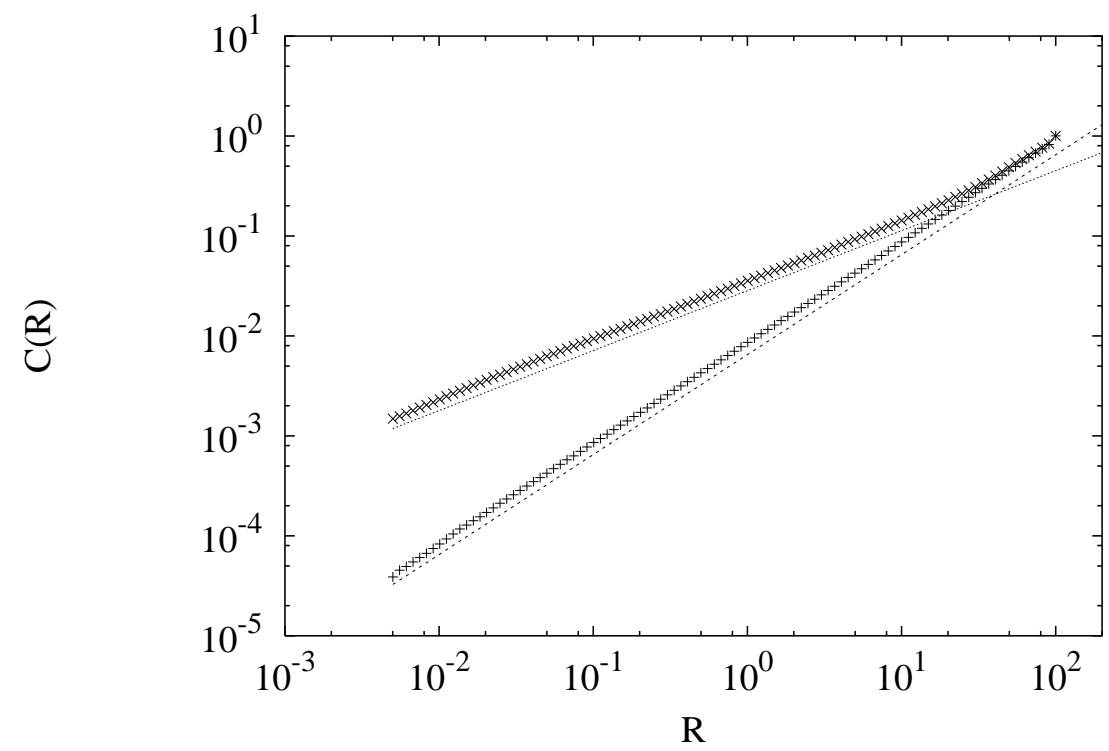

FIG. 5. $\mathrm{C}(\mathrm{R})$ vs. $\mathrm{R}$ for $\tau=100, r=0.6$ (top) and $\tau=100, r=0.99$ (bottom) with $N=200$. Correlation dimension takes respectively the values $d_{2}=0.59$ and $d_{2}=1$.

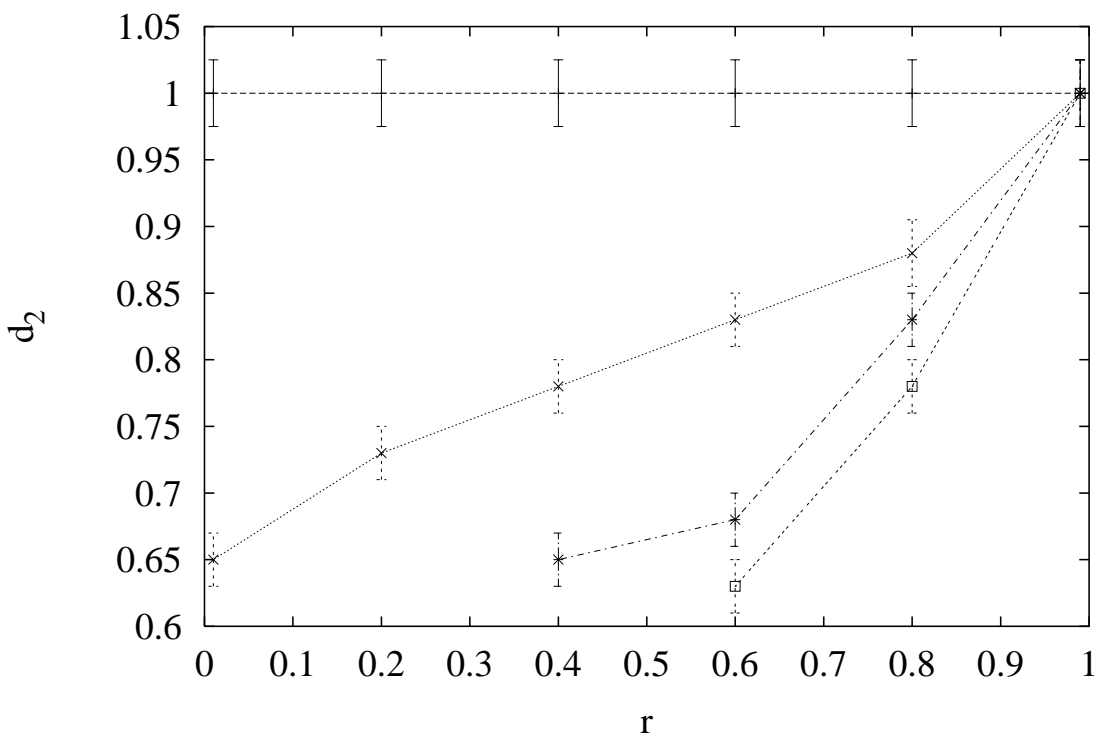

FIG. 6. The correlation dimension $d_{2}$ vs. $r$ for different values of $\tau$ : from top to bottom $\tau$ is $0.01,2,100$ and 1000. $N=200$ in all simulations 


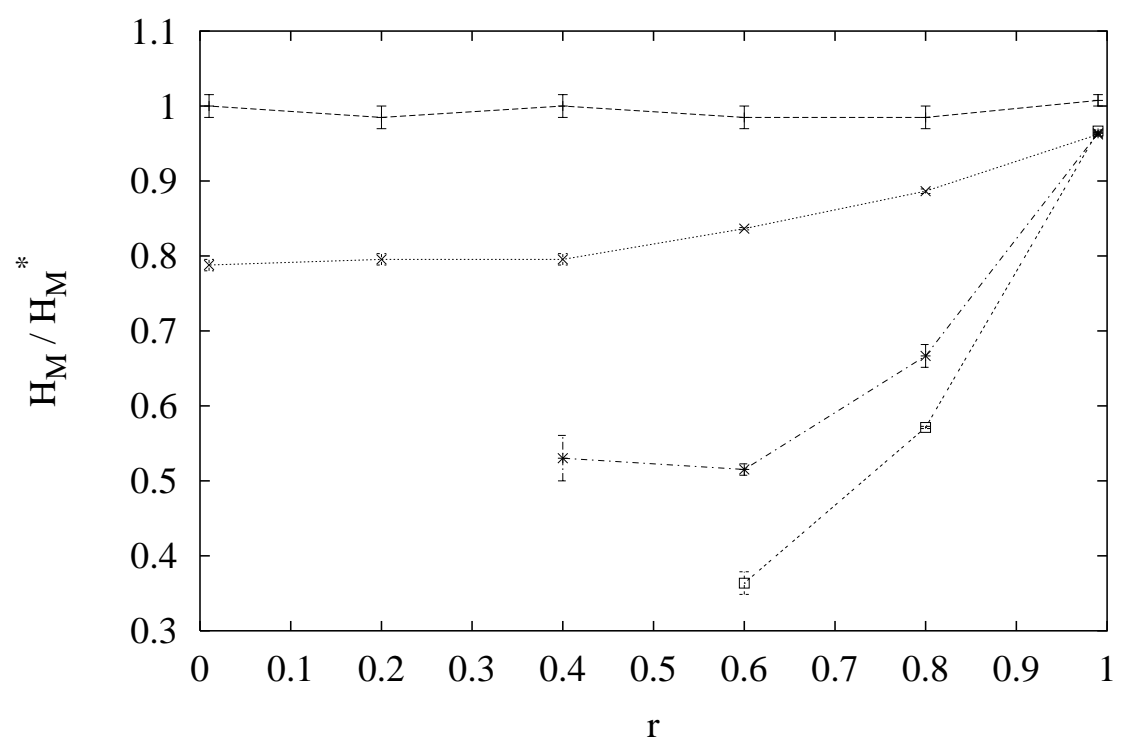

FIG. 7. $H_{M} / H_{M}^{*}$ vs. $r$ for different $\tau$ : form top to bottom $\tau$ is $0.01,2,100$ and 1000 , with $\mathrm{N}=200$ and $\mathrm{M}=80\left(H_{M}^{*} \approx 63\right)$

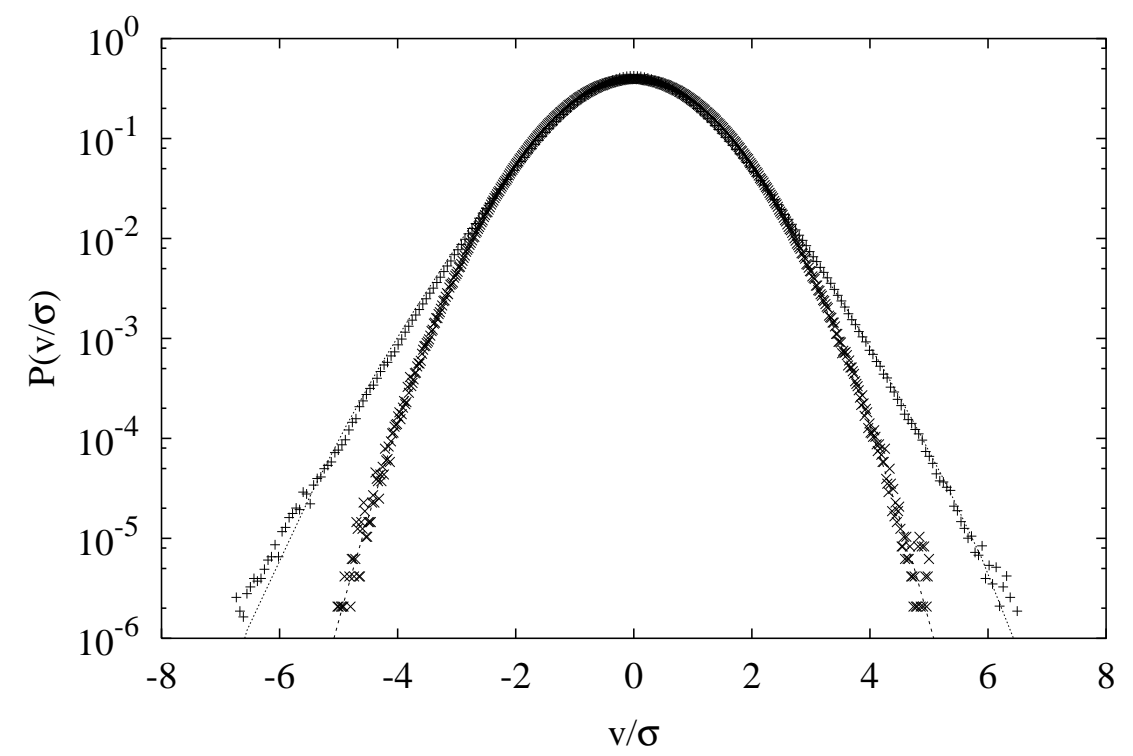

FIG. 8. The rescaled velocity distribution $P(v / \sigma)$ vs. $v / \sigma$. Pluses $(+)$ are data from simulation with $\tau=100, r=0.7$. Crosses are data with $\tau=0.01, r=0.99$. The dot-dashed line represents the gaussian distribution, while the dashed line represents the fit discussed in section $\mathrm{IV}$. 


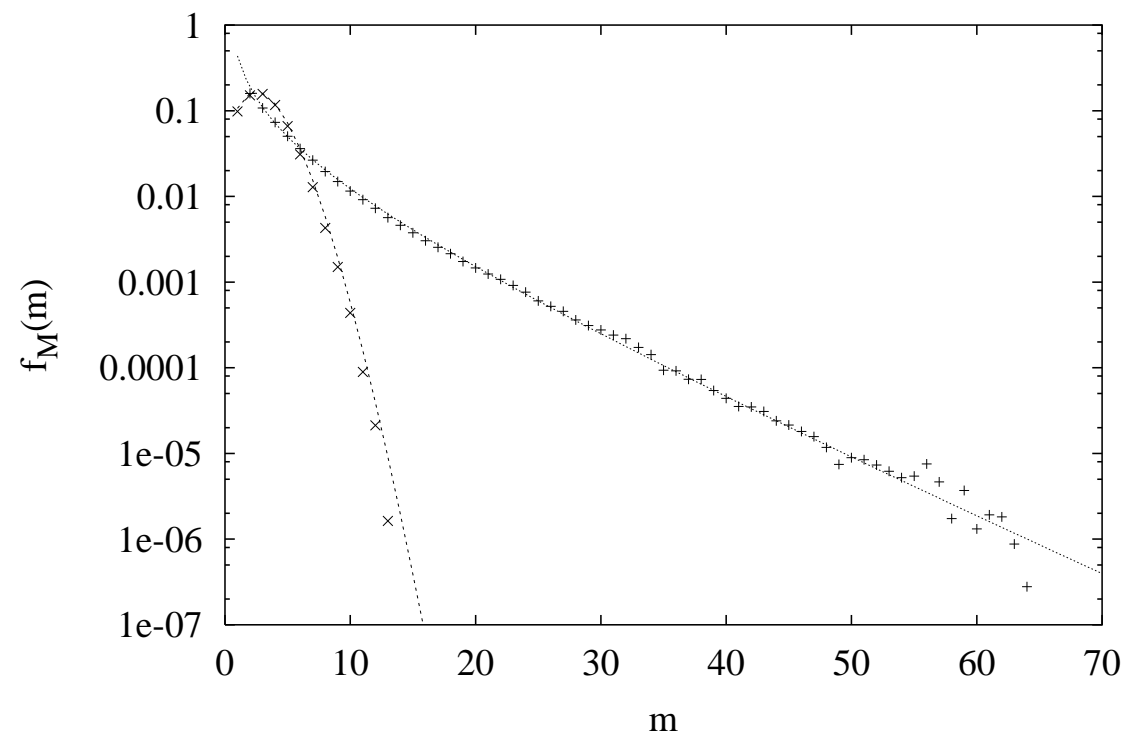

FIG. 9. Density distribution vs. $m$ for two different choices of parameters: $\tau=0.01, r=0.99$ and $\tau=100, r=0.7, N=300$ and $M=100$. The former is fitted by a Poisson distribution with $\lambda=3$, while the latter is fitted by $\frac{1}{m} e^{-0.14 * m}$

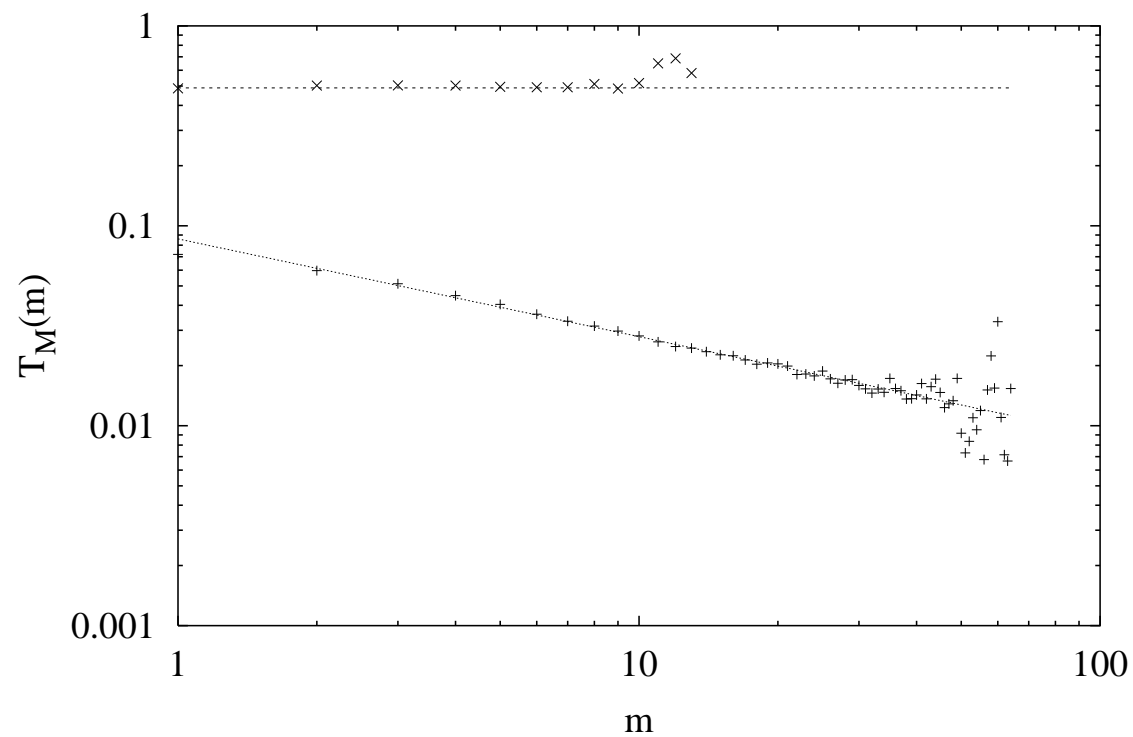

FIG. 10. Box granular temperature $T_{M}(m)$ against $m$, when $\tau=0.01, r=0.99$ and $\tau=100$, $r=0.7$ (in this case th fit $m^{-0.5}$ is plotted). 


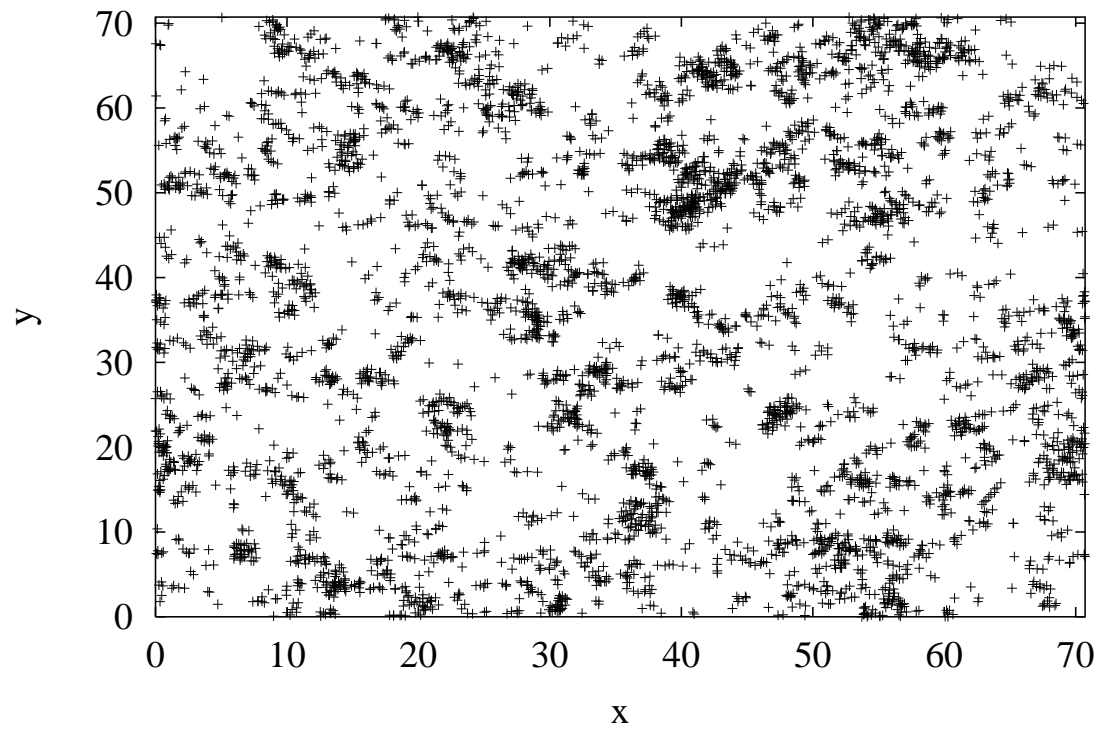

FIG. 11. Snapshot of particle distribution in 2 dimensions in the clusterized regime. $N=5000$, $\tau_{c}=0.5, l=0.63, \tau=100, r=0.01$.

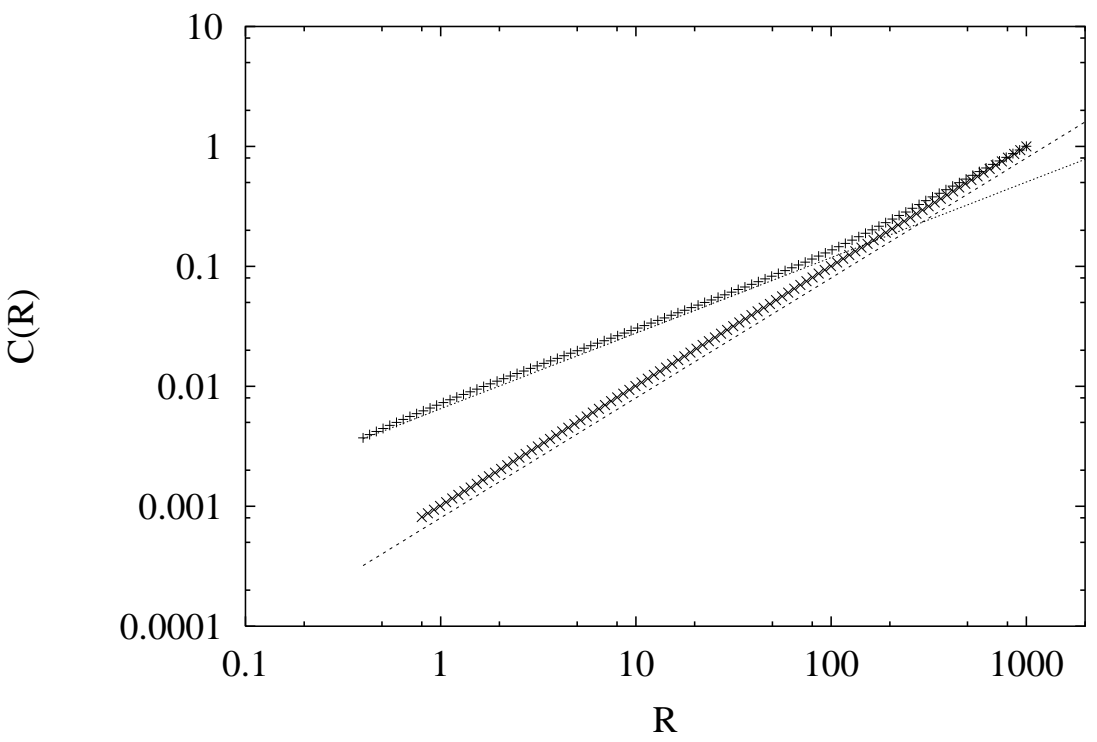

FIG. 12. $\mathrm{C}(\mathrm{R})$ vs. $\mathrm{R}$ in the one-dimensional system. $N=2000, \tau_{c}=0.5, l=0.4$. The top curve is for $\tau=100, r=0.5$, the bottom one is for $\tau=0.01, r=0.99$. Correlation dimension is, respectively, $d_{2}=0.55$ and $d_{2}=1$. 


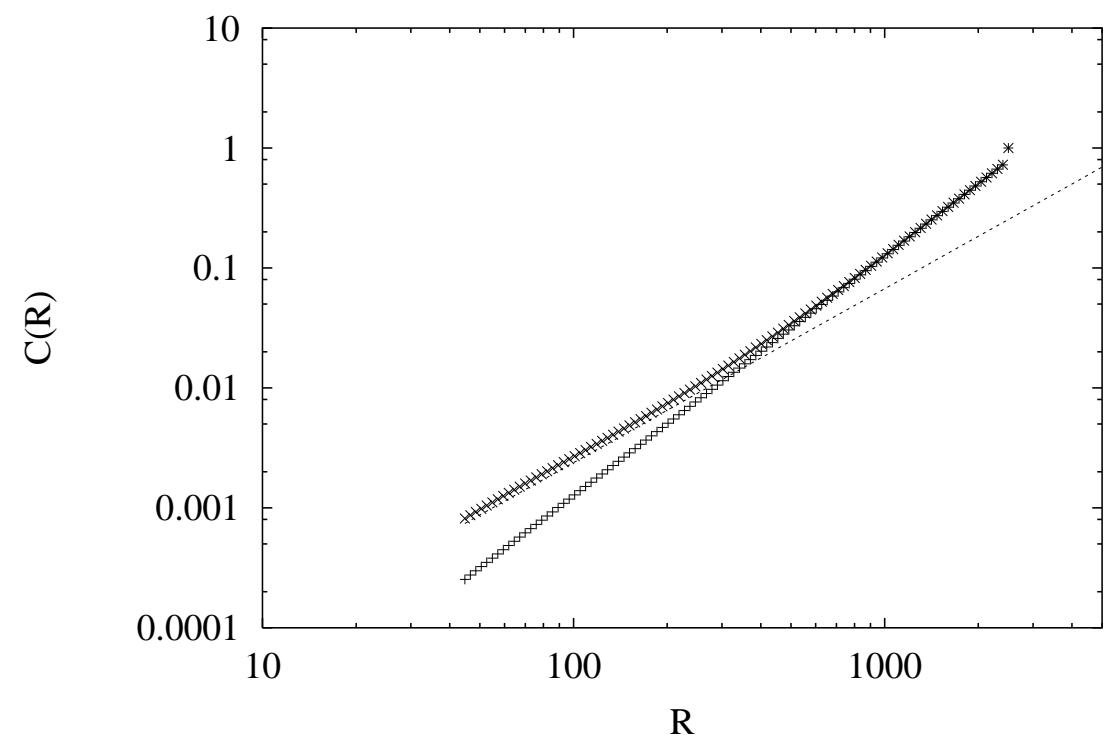

FIG. 13. $\mathrm{C}(\mathrm{R})$ vs. $\mathrm{R}$ in 2 -d. $N=5000, \tau_{c}=0.5, l=0.71$. Top curve is for $\tau=100, r=0.5$, while the bottom one is for $\tau=0.01, r=0.99$. The correlation dimension is $d_{2}=1.45$ and $d_{2}=2$, respectively
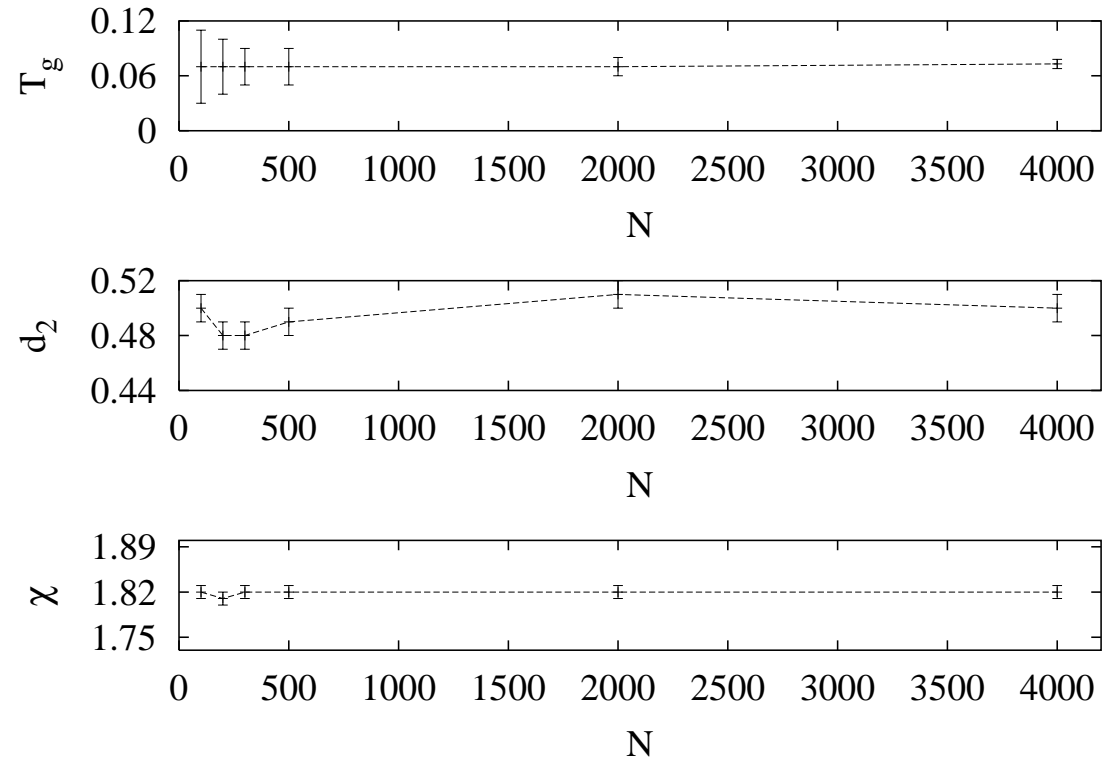

FIG. 14. The granular temperature $T_{g}$, fractal dimension $d_{2}$ and collision rate $\chi$ vs number of particle $N$, for the model in one dimension, with $\tau_{c}=0.5, l=0.4, \tau=100$ and $r=0.5$. 

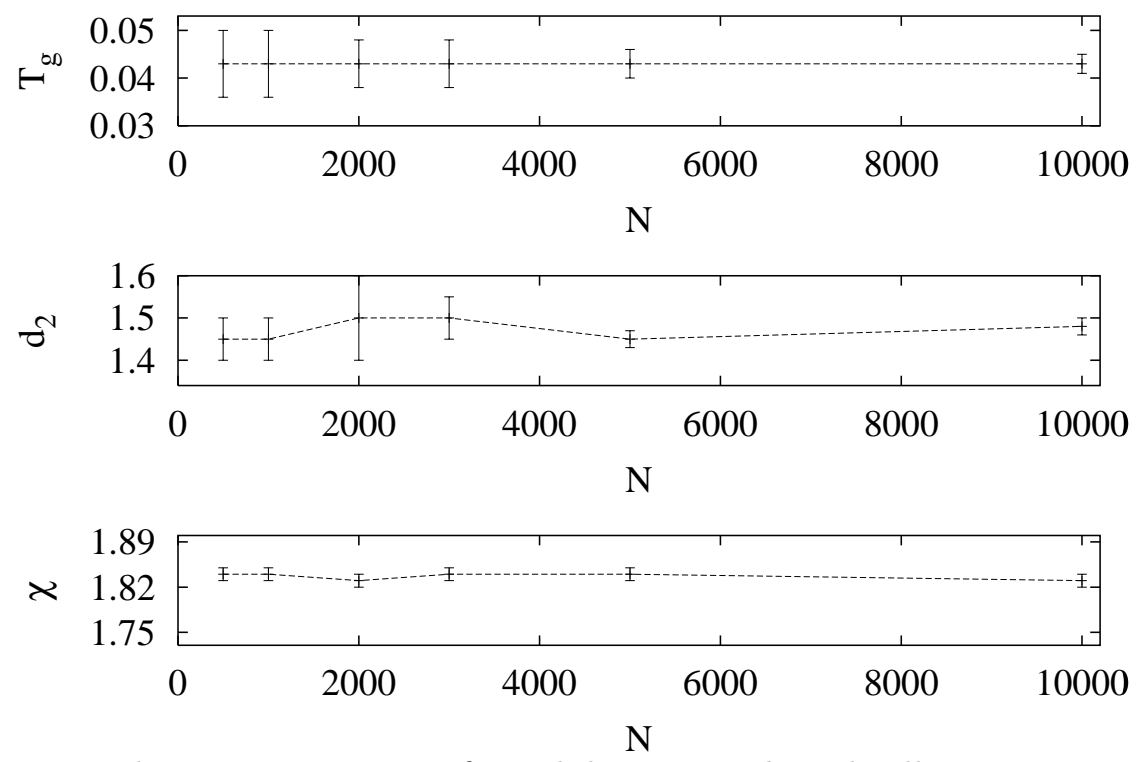

FIG. 15. The granular temperature $T_{g}$, fractal dimension $d_{2}$ and collision rate $\chi$ against number of particle $N$, for the model in two dimensions, with $\tau_{c}=0.5, l=0.63, \tau=100, r=0.5$
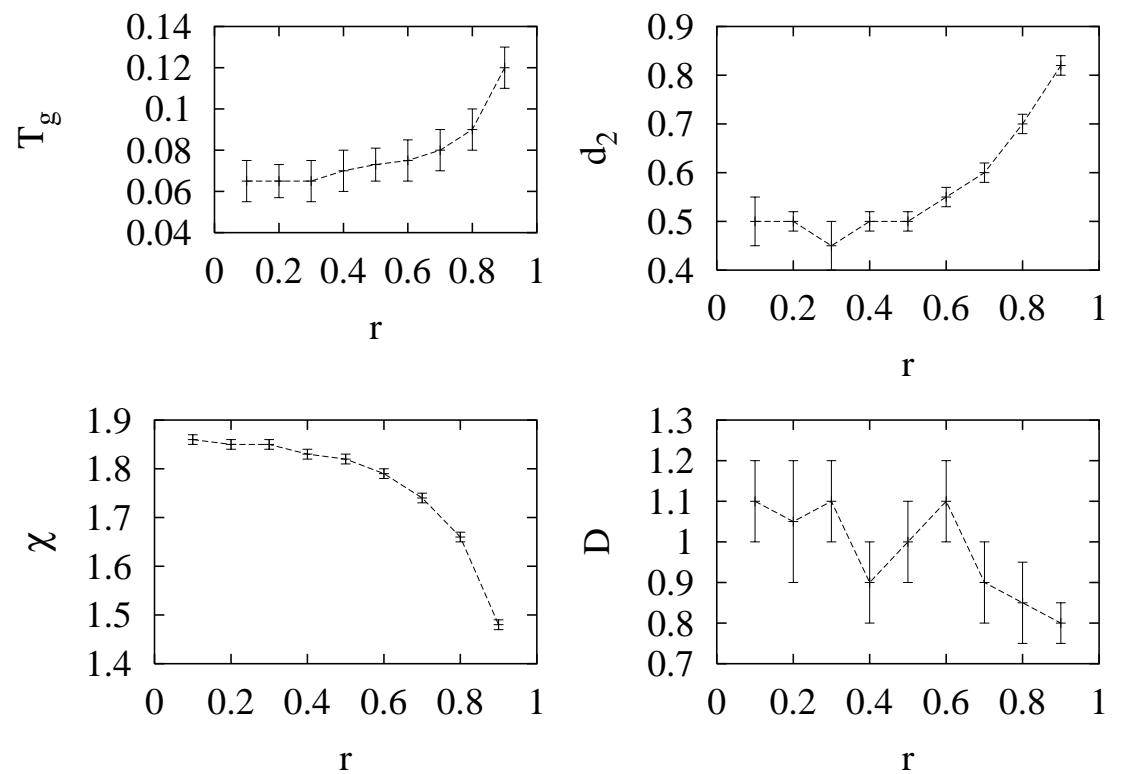

FIG. 16. The granular temperature $T_{g}$, fractal dimension $d_{2}$, collision rate $\chi$ and diffusion coefficient $D$ against restitution coefficient $r$, for the model in one dimension, with $N=4000$, $\tau_{c}=0.5, l=0.4, \tau=100$ and $r=0.5$. 

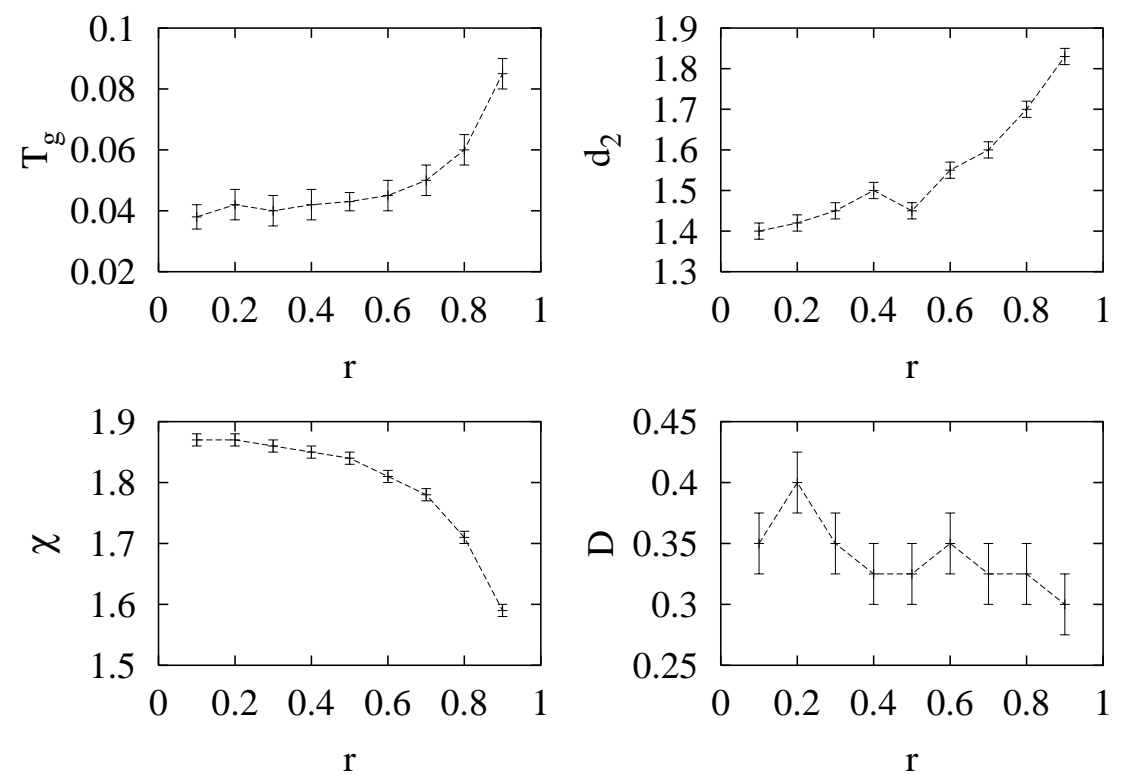

FIG. 17. The granular temperature $T_{g}$, fractal dimension $d_{2}$, collision rate $\chi$ and diffusion coefficient $D$ against restitution coefficient $r$, for the model in two dimensions, with $N=3000$, $\tau_{c}=0.5, l=0.63, \tau=100, r=0.5$

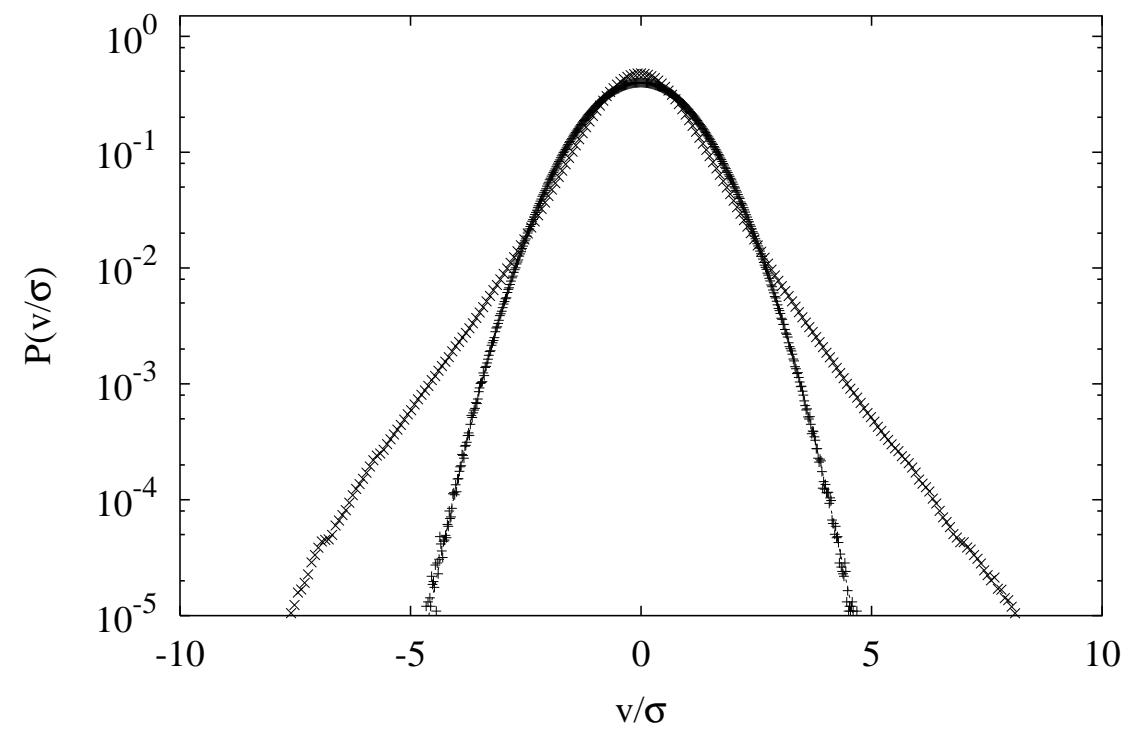

FIG. 18. Distribution of velocities in a gaussian $(\tau=0.01, r=0.99)$ and a non-gaussian regime $(\tau=100, r=0.5)$ for the one dimensional system. In both cases $N=2000, \tau_{c}=0.5, l=0.4$. The dashed curve represents the gaussian. 


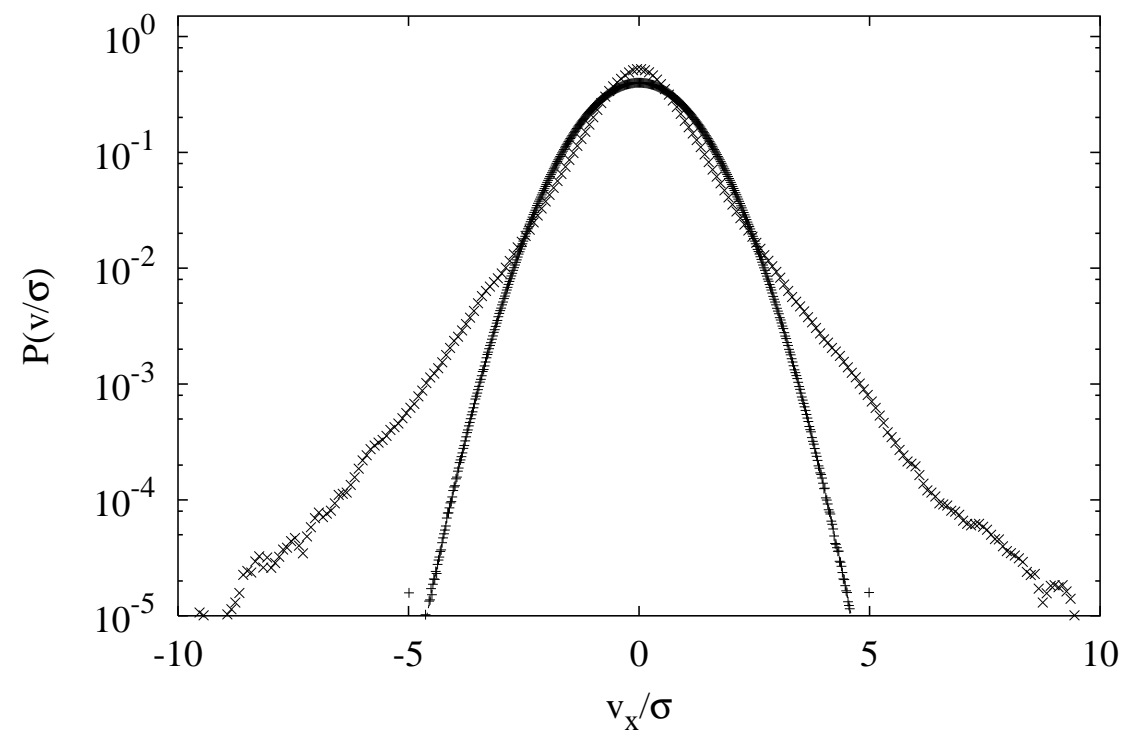

FIG. 19. Distribution of velocities in a gaussian $(\tau=0.01, r=0.99)$ and a non-gaussian regime $(\tau=100, r=0.5)$ for the two dimensions case. It is always $N=10000, \tau_{c}=0.05, l=0.22$ and the dashed line represents the gaussian

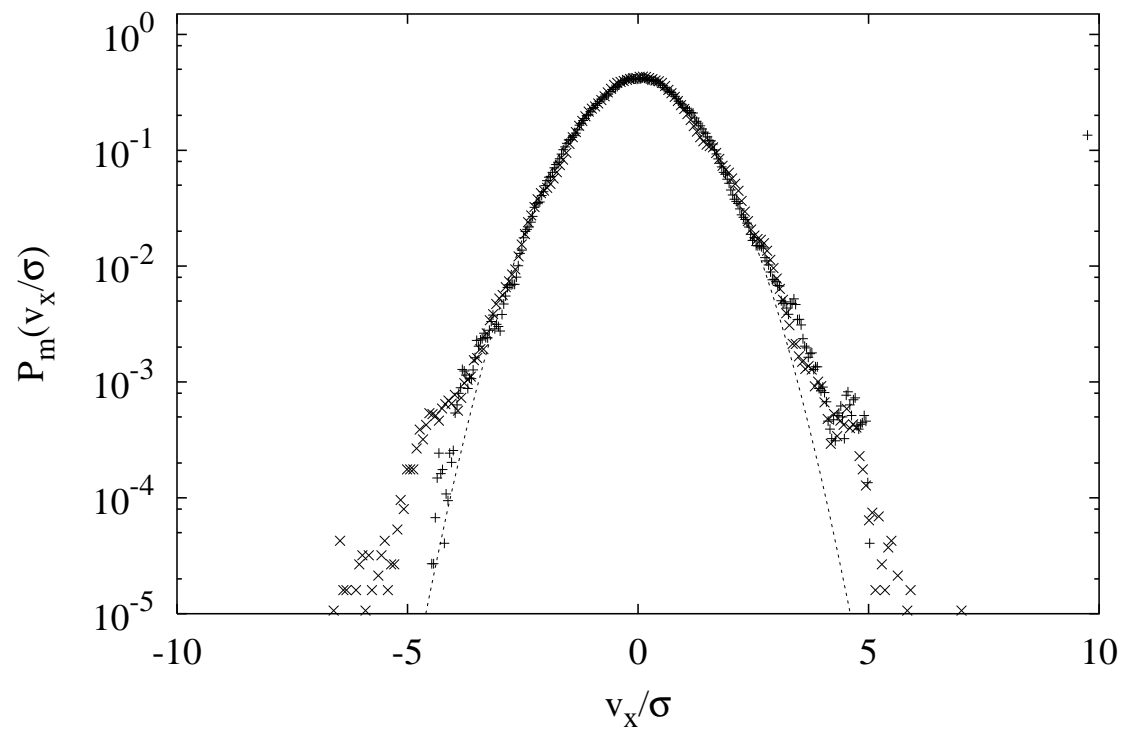

FIG. 20. Distribution of velocities restricted to number density $m=1$ (pluses ' + ') and $m=5$ (crosses 'X'), in a 2-dimensional case, with $N=10000, \tau_{c}=0.05, l=0.22, \tau=100, r=0.5$. 


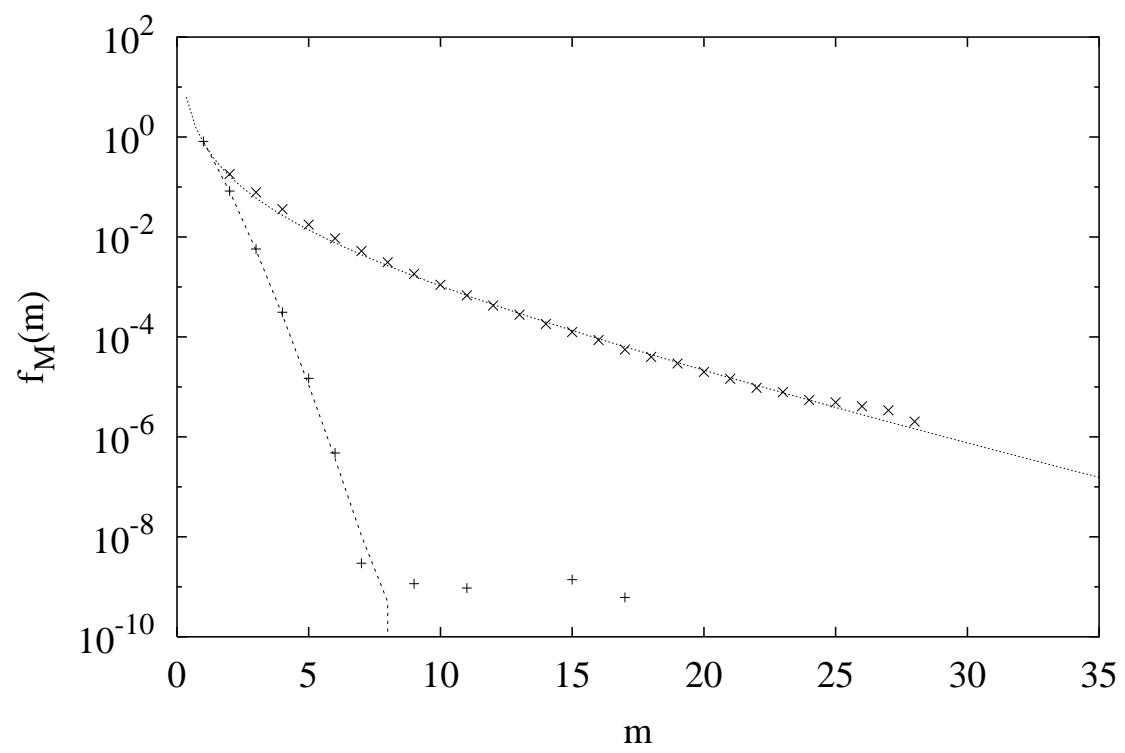

FIG. 21. Distribution density $f_{M}(m)$ vs. $m$ for two one-dimensional cases: $\tau=100, r=0.5$ and $\tau=0.01, r=0.99$. In both cases: $N=500, \tau_{c}=0.5, l=0.4, M=12000$. There are two curves superimposed: a Poisson function (with $\lambda=N / M \approx 0.04)$ and $m^{-1.95} \exp (-0.26 * m)$ fit for the clusterized regime.

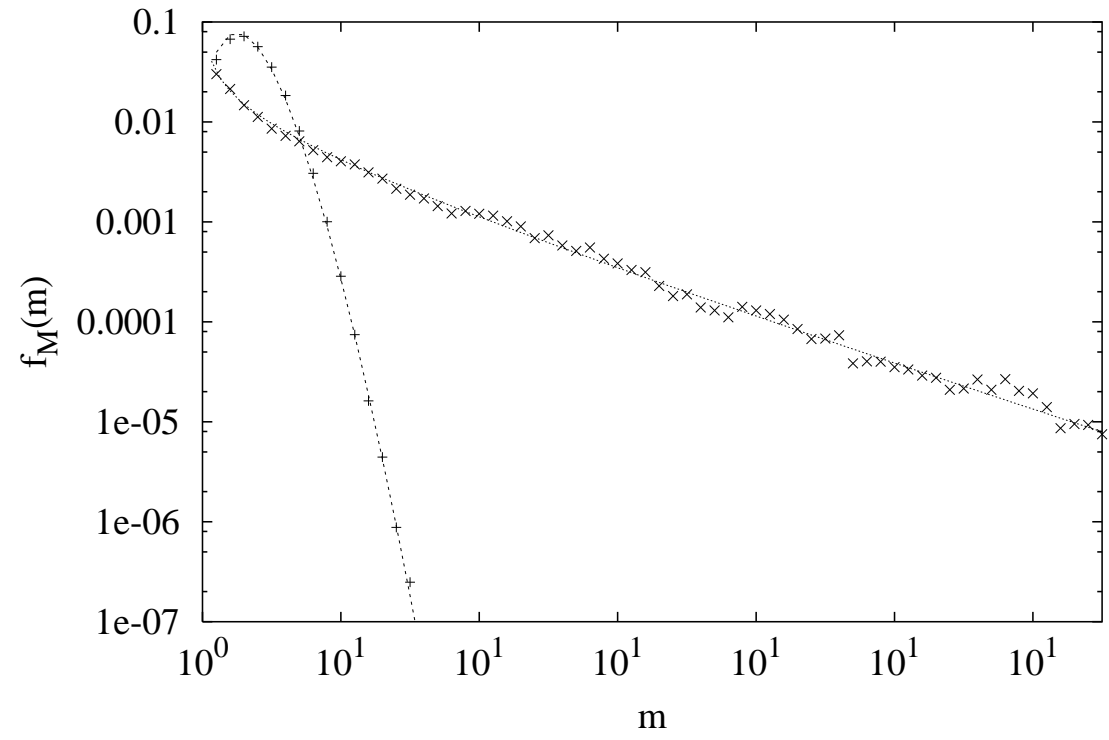

FIG. 22. Distribution density $f_{M}(m)$ vs. $m$ for two bidimensional cases: $\tau=100, r=0.5$ and $\tau=0.01, r=0.99$. In both cases: $N=10000, \tau_{c}=0.05, l=0.22, M=3200$. There are two curves superimposed: a Poisson function (with $\lambda=N / M=3.125)$ and $m^{-0.5} \exp (-0.097 * m)$ fit for the clusterized regime. 


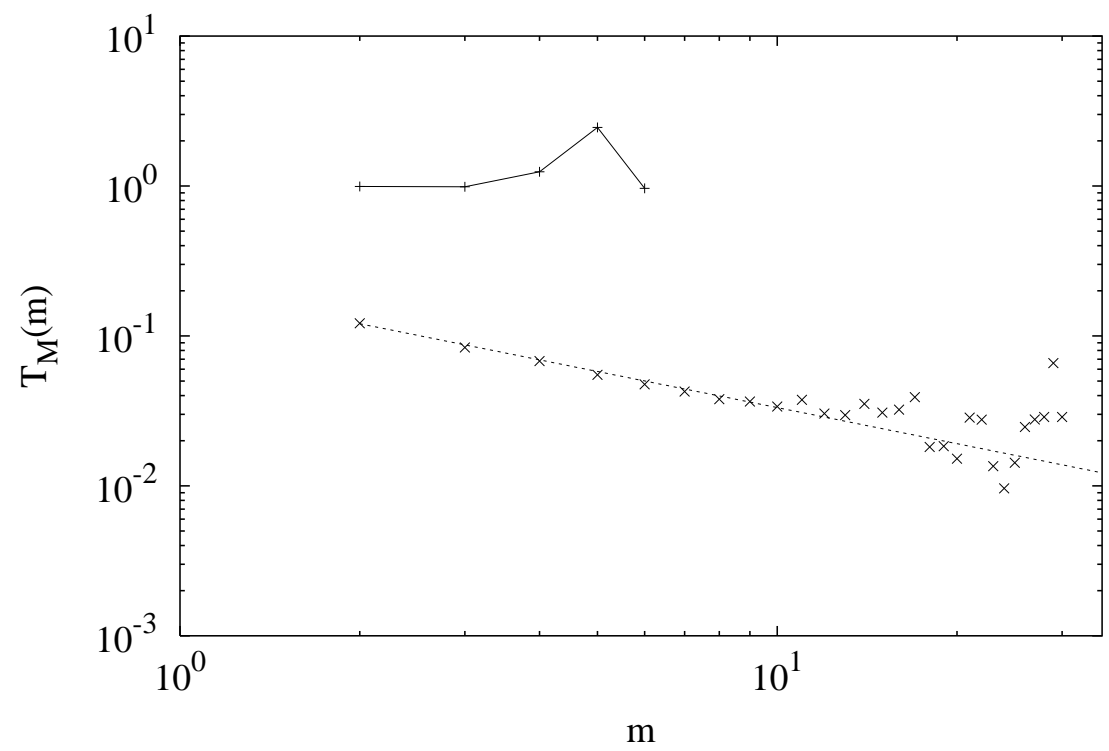

FIG. 23. Box granular temperature $T_{M}(m)$ vs. $m$ for two one-dimensional cases: $\tau=100$, $r=0.5$ and $\tau=0.01, r=0.99$. In both cases: $N=500, \tau_{c}=0.5, l=0.4, M=12000$. The gaussian case is constant, while the non-gaussian case is fitted by $\sim m^{-0.8}$.

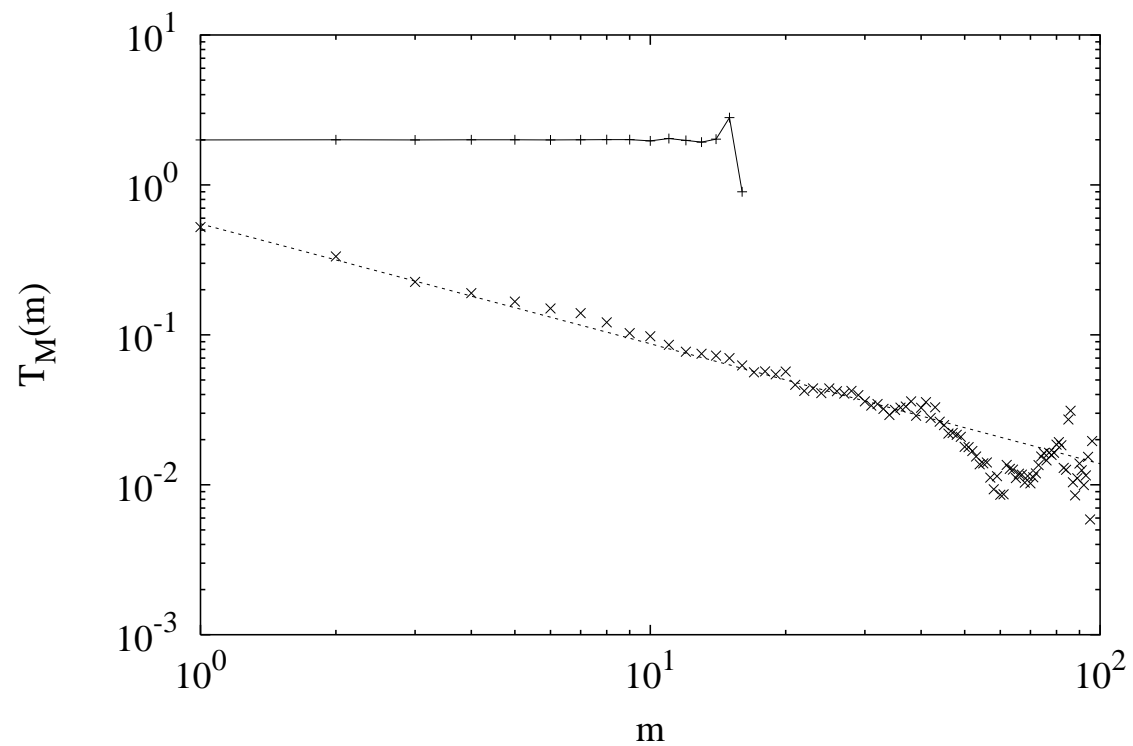

FIG. 24. Box granular temperature $T_{M}(m)$ vs. $m$ for two bidimensional cases: $\tau=100, r=0.5$ and $\tau=0.01, r=0.99$. In both cases: $N=10000, \tau_{c}=0.05, l=0.22, M=3200$. The gaussian case is constant, while the non-gaussian one is fitted by $\sim m^{-0.8}$. 


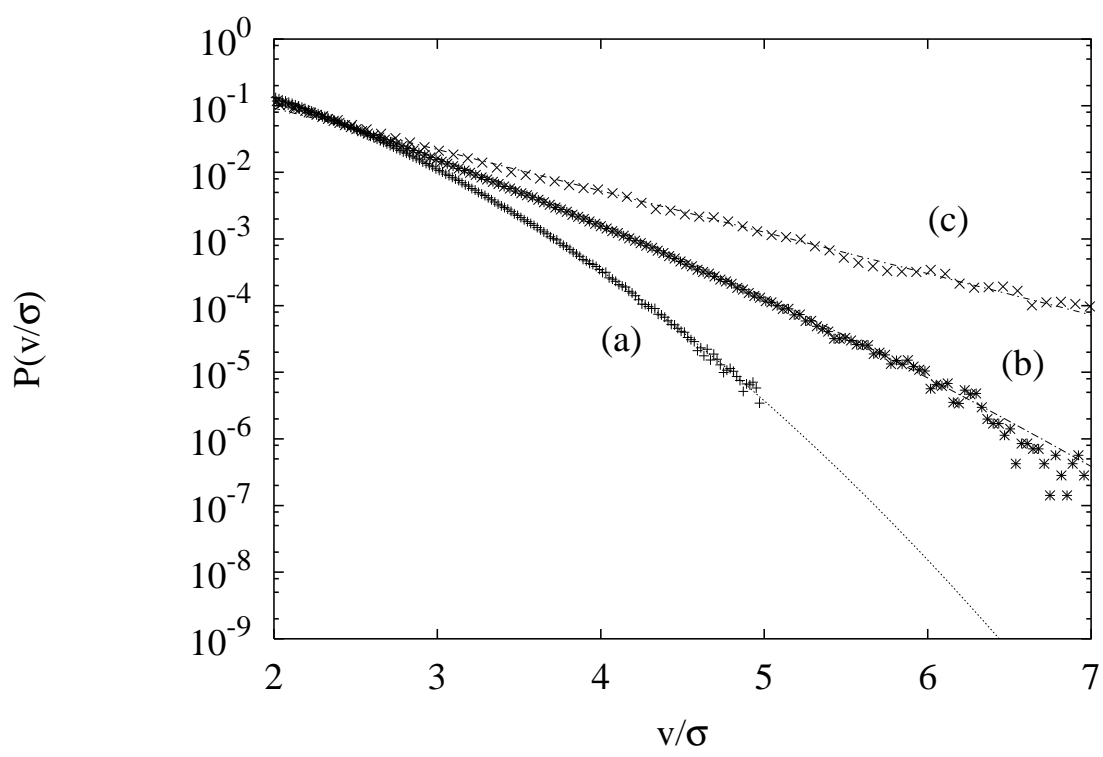

FIG. 25. Rescaled distributions of velocities (particular) for three different choices of parameters, in two dimensions: (a) $N=10000, \tau=0.01, r=0.99$, with Gaussian fit; (b) $N=3000$, $\tau=5, r=0.5$ with the fit $\sim \exp \left(-v^{3 / 2} / 1.25\right)$; (c) $N=10000, \tau=100, r=0.2$ with the fit $\sim \exp (-v / 0.7)$. In the cases (a) and (c): $\tau_{c}=0.05, l=0.22$. In the case (b): $\tau_{c}=0.5$ and $l=0.63$. 